\title{
The ratio $F_{2}^{\mathrm{n}} / F_{2}^{\mathrm{p}}$ in deep inelastic muon scattering
}

\author{
The New Muon Collaboration (NMC)
}

P. Amaudruz ${ }^{13 a}$, M. Arneodo ${ }^{14}$, A. Arvidson ${ }^{15}$, B. Badelek ${ }^{17,++}$, G. Baum ${ }^{1, *}$, J. Beaufays ${ }^{9 b, * *}$, I.G. Bird ${ }^{4 c, *}$, M. Botje ${ }^{13}$, C. Broggini ${ }^{8 d}$, W. Brückner ${ }^{4, *}$,

A. Brüll ${ }^{3, *}$, W.J. Burger ${ }^{13 e}$, J. Ciborowski ${ }^{9 f, * *}$, R. van Dantzig ${ }^{9, * *}$, H. Döbbeling ${ }^{4 \mathrm{~g}, *}$, J. Domingo ${ }^{13 \mathrm{~h}}$, J. Drinkard ${ }^{12}$, H. Engelien ${ }^{3, *}$, M.I. Ferrero ${ }^{14}$,

L. Fluri ${ }^{8}$, P. Grafstrom ${ }^{15 i}$, D. von Harrach ${ }^{4 j}$.* , M. van der Heijden ${ }^{9, * *}$,

C. Heusch ${ }^{12}$, Q. Ingram ${ }^{13}$, K. Janson ${ }^{15}$, M. de Jong ${ }^{9, * *}$, E.M. Kabuss ${ }^{4 j, *}$,

R. Kaiser ${ }^{3, *}$, T.J. Ketel ${ }^{9, * *}$, F. Klein ${ }^{6, *}$, B. Korzen ${ }^{18, *}$, U. Krüner ${ }^{18, *}$, S. Kullander ${ }^{15}$, U. Landgraf ${ }^{3, *}$, F. Lettenström ${ }^{12}$, T. Lindqvist ${ }^{15}$, G.K. Mallot ${ }^{6, *}$, C. Mariotti ${ }^{14}$, G. van Middelkoop ${ }^{2,9, * *}$, A. Milsztajn ${ }^{11}$, Y. Mizuno ${ }^{4 k, *}$, J. Nassalski ${ }^{16,9,+, * *}$, D. Nowotny ${ }^{4, *}$, N. Pavel ${ }^{18 \ell, *}$, C. Peroni ${ }^{14}$,

H. Peschel ${ }^{18 \mathrm{~m}, *}$, B. Povh ${ }^{4,5} *$, R. Rieger ${ }^{6, *}$, K. Rith ${ }^{4, *}$, K. Röhrich ${ }^{6 n, *}$, E. Rondio ${ }^{17,++}$, L. Ropelewski ${ }^{17,++}$, A. Sandacz ${ }^{16,+}$, C. Scholz ${ }^{4, *}$, R. Schumacher ${ }^{130}$, U. Sennhauser ${ }^{13 p}$, F. Sever ${ }^{1 \mathrm{q}, *}$, T.-A. Shibata ${ }^{5, *}$, M. Siebler ${ }^{1, *}$, A. Simon ${ }^{4, *}$, A. Staiano ${ }^{14}$, G. Taylor ${ }^{10 r}$, M. Treichel ${ }^{4 s, *}$, M. Virchaux ${ }^{11}$, J.L. Vuilleumier ${ }^{8}$, T. Walcher ${ }^{6, *}$, R. Windmolders ${ }^{7}$ and F. Zetsche ${ }^{4, *}$

${ }^{1}$ Bielefeld University, Physics Department, 4800 Bielefeld, Germany

${ }^{2}$ CERN, 1211 Geneva 23, Switzerland

* Supported by Bundesministerium für Forschung und Technologie.

** Supported in part by FOM, Vrije Universiteit Amsterdam and NWO.

+ Supported by CPBP.01.06.

++ Supported by CPBP.01.09.

a Now at TRIUMF, Vancouver, BC V6T 2A3, Canada.

b Now at Trasys, 1930 Zaventem, Belgium.

c Now at NIKHEF-K, P.O. Box 4395, 1009 AJ Amsterdam, The Netherlands.

¿ Now at INFN, Laboratori Nazionali del Gran Sasso, 67010 Assergi, Italy.

e Now at Université de Genève, 1211 Geneva 4, Switzerland.

f Now at University of Warsaw, 00681 Warsaw, Poland.

g Now at GSI, 6100 Darmstadt, Germany.

h Now at CEBAF, Newport News, VA 23606, USA.

i Now at CERN, 1211 Geneva 23, Switzerland.

j Now at Mainz University, 6500 Mainz, Germany.

k Now at Osaka University, Osaka 567, Japan.

${ }^{\ell}$ Now at DESY, 2000 Hamburg 52, Germany.

${ }^{m}$ Now at Gruner and Jahr AG\&CoKG, 2210 Itzehoe, Germany.

${ }^{n}$ Now at IKP2-KFA, 5170 Jülich, Germany.

- Now at Carnegie Mellon University, Pittsburgh, PA 15213, USA. 


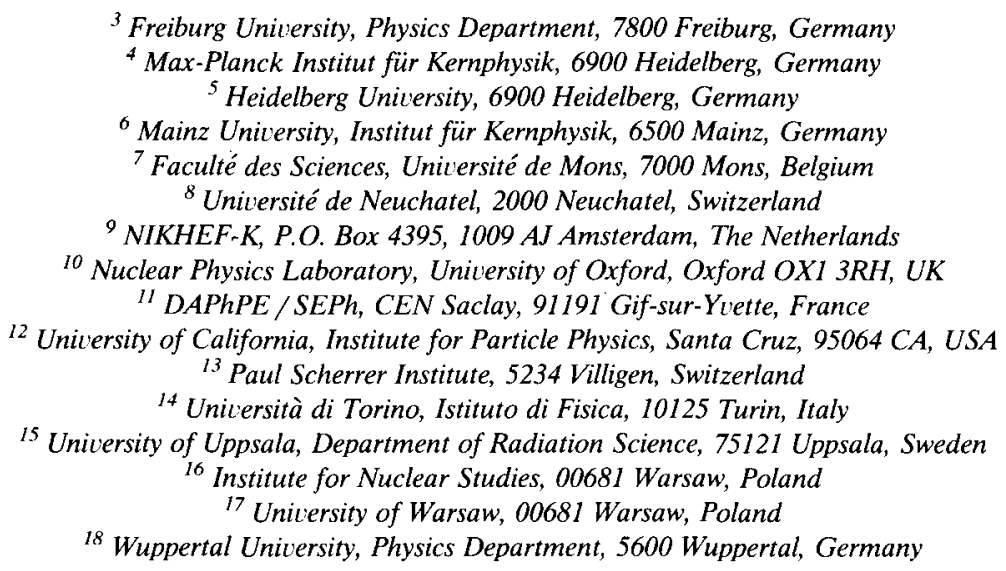

Received 7 October 1991

Accepted for publication 17 October 1991

\begin{abstract}
Results are presented on the ratio of neutron and proton structure functions, $F_{2}^{\text {n }} / F_{2}^{\text {p }}$, deduced from deep inelastic scattering of muons from hydrogen and deuterium. The data, which were obtained at the CERN muon beam at 90 and $280 \mathrm{GeV}$ incident energy, cover the kinematic range $x=0.002-0.80$ and $Q^{2}=0.1-190 \mathrm{GeV}^{2}$. The measured structure function ratios have small statistical and systematic errors, particularly at small and intermediate $x$. The observed $Q^{2}$ dependence in the range $x=0.1-0.4$ is stronger than predicted by perturbative QCD. From the present data together with results from other experiments it is suggested that the twist-four coefficient for the proton is smaller than that for the neutron for $x$ larger than 0.2 .
\end{abstract}

\title{
1. Introduction
}

In the quark-parton model, the ratio of the neutron and proton structure functions, $F_{2}^{\mathrm{n}} / F_{2}^{\mathrm{p}}$, is related to the ratio of the down- and up-quark momentum distributions. Accurate measurements of $F_{2}^{\mathrm{n}} / F_{2}^{\mathrm{p}}$ put strong constraints on parton distributions. Precise knowledge of these distributions, particularly in the low- $x$ region, is important in calculating reliably hard scattering cross sections in $p p, p \bar{p}$ and ep collisions. In addition this ratio can be used to measure the Gottfried sum and to set a constraint on the onset of shadowing in deuterium.

The $Q^{2}$ dependence of the structure function ratio provides a test of perturbative QCD. Logarithmic variation with $Q^{2}$ (scale breaking) of the nucleon structure function $F_{2}\left(x, Q^{2}\right)$ can be calculated in the framework of perturbative QCD up to next-to-leading order in the strong coupling constant $\alpha_{\mathrm{s}}$ [1]. Due to the different

P Now at EMPA, 8600 Dubendorf, Switzerland.

q On leave from Jozef Stefan Institut, Ljubljana 61111, Yugoslavia, now at DAPNIA Saclay, 91191 Gif-sur-Yvette, France.

r Now at University of Melbourne, Parkville, Victoria 3052, Australia.

s Now at Université de Neuchatel, 2000 Neuchatel, Switzerland. 
flavour composition of the proton and neutron, the $Q^{2}$ dependences are slightly different in $F_{2}^{\mathrm{n}}$ and $F_{2}^{\mathrm{p}}$ and give rise to a small, calculable scale breaking in the ratio $F_{2}^{\mathrm{n}} / F_{2}^{\mathrm{p}}$. In addition, non-logarithmic contributions to the scale breaking are due to the interaction of the struck quark with the spectator quarks (higher-twist effects) and to target mass effects. Whereas target mass effects can be rigorously treated in a QCD analysis of structure functions, the magnitude of $1 / Q^{2 n-2}$ contributions from twist $-2 n$ operators is more difficult to estimate and cannot be calculated in a model-independent way [2]. Experimental data on the $Q^{2}$ dependence of the ratio $F_{2}^{\mathrm{n}} / F_{2}^{\mathrm{p}}$ combined with predictions from perturbative QCD can be used to determine the difference between higher-twist terms in the proton and neutron. A recent analysis of the SLAC and BCDMS data may indicate such differences [3].

Results on the $x$ dependence of $F_{2}^{\mathrm{n}} / F_{2}^{\mathrm{p}}$ were published by the EMC [4] and SLAC [5-7]. Higher precision data on the $x$ and $Q^{2}$ dependence come from the BCDMS collaboration [8] and from a reanalysis of SLAC electron scattering data [9]; both experiments cover a kinematic range down to $x=0.06$, and $0.5<Q^{2}<30$ $\mathrm{GeV}^{2}$ (SLAC) and $8<Q^{2}<260 \mathrm{GeV}^{2}$ (BCDMS). In all these experiments $F_{2}^{\text {n }} / F_{2}^{\text {p }}$ was determined from separate measurements of the structure functions $F_{2}^{\mathrm{p}}$ and $F_{2}^{\text {d }}$.

In the present experiment (NMC; CERN-NA37) the ratio $F_{2}^{\mathrm{n}} / F_{2}^{\mathrm{p}}$ was obtained from simultaneous measurements on hydrogen and deuterium at incident muon energies of 90 and $280 \mathrm{GeV}$ using a symmetric target arrangement. This reduces systematic errors due to the spectrometer acceptance and normalisation and allows the measurement to be extended reliably to kinematic regions where the detector acceptance is small. The data cover a broad kinematic range of $0.002<x<0.80$ and $0.1<Q^{2}<190 \mathrm{GeV}^{2}$ with systematic errors typically below $1 \%$. First results from part of these data were published in refs. [10,11].

This paper is organised as follows: In sect. 2, the method used to measure structure function ratios is outlined. The NMC spectrometer is briefly described in sect. 3. The analysis of the data, including the event reconstruction and selection, consistency checks and corrections to the data are treated in detail in sect. 4 . In sects. 5 and 6 , the results are presented and the $Q^{2}$ dependence is interpreted in terms of higher-twist effects.

\section{The method}

In the one-photon exchange approximation (fig. 1) the differential cross section per nucleon for deep inelastic charged lepton scattering on an unpolarised target is related to the structure function $F_{2}\left(x, Q^{2}\right)$ and $R\left(x, Q^{2}\right)$ by

$$
\frac{\mathrm{d}^{2} \sigma^{1 \gamma}}{\mathrm{d} x \mathrm{~d} Q^{2}}=\frac{4 \pi \alpha^{2} F_{2}\left(x, Q^{2}\right)}{Q^{4} x}\left[1-y-\frac{Q^{2}}{4 E^{2}}+\frac{y^{2}+Q^{2} / E^{2}}{2\left(1+R\left(x, Q^{2}\right)\right)}\right],
$$




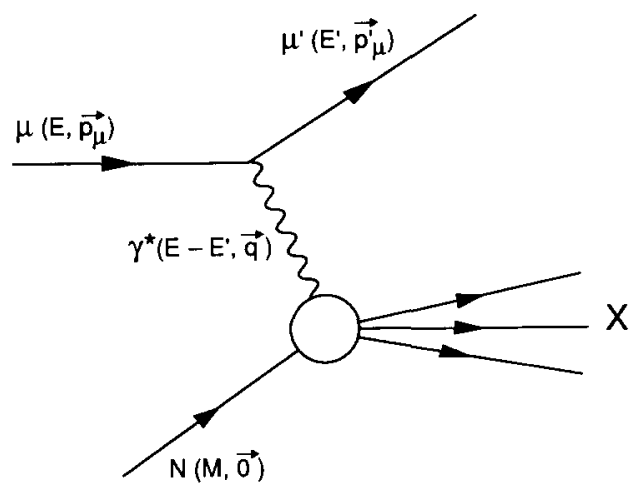

Fig. 1. Diagram for deep inelastic scattering of a muon from a nucleon in the one-photon exchange approximation.

where $E$ is the incoming lepton energy, $-Q^{2}$ the square of the four-momentum transfer, $x=Q^{2} / 2 M \nu$ the Bjorken scaling variable, $\nu$ the energy transfer, $y=\nu / E$ and $M$ the proton mass. The function $R\left(x, Q^{2}\right)$ is the ratio of longitudinally to transversely polarised virtual photon absorption cross sections.

The ratio of cross sections on hydrogen and deuterium was measured with the target arrangement shown in fig. 2. Two sets of targets (labelled 1 and 2 in fig. 2) were alternately moved into the beam every half hour. Each set consisted of two target vessels of equal length situated one behind the other along the beam line. The upstream (downstream) vessel of target set 1 was filled with liquid deuterium (hydrogen). The sequence of target materials was reversed in target set 2 .

The number of scattered muons detected in the spectrometer and originating in e.g. the upstream deuterium target is given by

$$
N_{\mathrm{d}}^{\mathrm{up}}=\Phi_{1} \rho_{\mathrm{d}} \sigma_{\mathrm{d}} A_{\mathrm{d}}^{\mathrm{up}}
$$

\section{Upstream Downstream}

Set 1

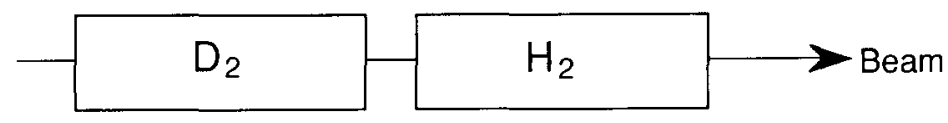

Set 2
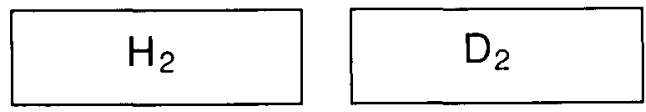

Fig. 2. Arrangement of the liquid hydrogen and deuterium targets. The sets 1 and 2 were alternately positioned in the muon beam. 
Here $\Phi_{1}$ is the integrated beam flux illuminating the targets of set $1, \rho_{\mathrm{d}}$ the number of target nucleons per unit area, $\sigma_{\mathrm{d}}$ the cross section per nucleon for the inclusive process $\mu+\mathrm{d} \rightarrow \mu+\mathrm{X}$ and $A_{\mathrm{d}}^{\mathrm{up}}$ the acceptance of the detector for events originating from the upstream deuterium target. With similar expressions for muons scattered in the other three targets and the assumption that $A_{\mathrm{d}}^{\text {up }}=A_{\mathrm{p}}^{\text {up }}$ $=A^{\mathrm{up}}$ and $A_{\mathrm{d}}^{\mathrm{dn}}=A_{\mathrm{p}}^{\mathrm{dn}}=A^{\mathrm{dn}}$, one obtains

$$
\frac{\sigma_{\mathrm{d}}}{\sigma_{\mathrm{p}}}=\kappa \sqrt{\frac{N_{\mathrm{d}}^{\mathrm{up}} N_{\mathrm{d}}^{\mathrm{dn}}}{N_{\mathrm{p}}^{\mathrm{up}} N_{\mathrm{p}}^{\mathrm{dn}}}}
$$

with $\kappa=\rho_{\mathrm{p}} / \rho_{\mathrm{d}}$. In this way the ratio is calculated from the numbers of events only and does not depend on flux or acceptance.

Radiative corrections are taken into account by weighting each event with the ratio $\sigma^{1 \gamma} / \sigma$ and replacing the number of events in eq. (3) by the accumulated weights to obtain the cross section ratio $\sigma_{\mathrm{d}}^{1 \gamma} / \sigma_{\mathrm{p}}^{1 \gamma}$. Details on the calculation of these radiative corrections are given in subsect. 4.5 .

With the assumption that $R$ does not depend on the target nucleus, eq. (1) gives $F_{2}^{\mathrm{d}} / F_{2}^{\mathrm{p}}=\sigma_{\mathrm{d}}^{1 \gamma} / \sigma_{\mathrm{p}}^{1 \gamma}$. This assumption is supported by several experimental results: at low $Q^{2}$ from SLAC [12] in the range $0.10<x<0.86$ and $0.6<Q^{2}<20 \mathrm{GeV}^{2}$ and at high $Q^{2}$ by BCDMS [18] for $x>0.07$ and $Q^{2}>8 \mathrm{GeV}^{2}$. Then the structure function ratio $F_{2}^{\mathrm{n}} / F_{2}^{\mathrm{p}}$ is given by

$$
\frac{F_{2}^{\mathrm{n}}}{F_{2}^{\mathrm{p}}}=2 \frac{F_{2}^{\mathrm{d}}}{F_{2}^{\mathrm{p}}}-1=2 \frac{\sigma_{\mathrm{d}}^{1 \gamma}}{\sigma_{\mathrm{p}}^{1 \gamma}}-1
$$

Here nuclear effects, in particular Fermi motion in deuterium, have been neglected.

\section{The apparatus}

\subsection{INTRODUCTION}

The experiment was performed at the muon beam line M2 of the SPS at CERN with the upgraded EMC spectrometer shown in fig. 3. The incident muons had mean energies of 89 and $274 \mathrm{GeV}$ (nominal values 90 and $280 \mathrm{GeV}$ ) and an r.m.s. energy spread of $4 \%$. The beam intensities were $2 \times 10^{7} \mathrm{~s}^{-1}$ and $10^{7} \mathrm{~s}^{-1}$ during the $2 \mathrm{~s}$ spills of the SPS. The integrated beam fluxes amounted to $0.46 \times 10^{12}$ muons (14 days of data taking at $90 \mathrm{GeV}$ ) and $2.73 \times 10^{12}$ muons ( 83 days at 280 $\mathrm{GeV})$. 
The important modifications of the EMC apparatus [13] include: the complementary target designed to measure structure function ratios with small systematic uncertainties, the addition of new proportional chambers which improved the reconstruction efficiency and vertex resolution, a small-angle physics trigger, an upgrade of the data acquisition system including event buffering to reduce dead times, and a beam momentum calibration spectrometer.

\subsection{THE TARGETS}

The complementary target set-up shown in fig. 2 consisted of two sets of $3 \mathrm{~m}$ long liquid hydrogen and $3 \mathrm{~m}$ long liquid deuterium targets. The target vessels were made of mylar and had a diameter of $10 \mathrm{~cm}$. They were placed in vacuum tight hard paper containers with a diameter of $30 \mathrm{~cm}$. The beam, which had horizontal and vertical dimensions of 1.3 and $1.0 \mathrm{~cm}$ (r.m.s.) at the upstream target position was well contained in the targets over the entire length of the set-up. The target thicknesses were 21.06(1) $\mathrm{g} / \mathrm{cm}^{2}$ for $\mathrm{H}_{2}$ and $48.58(1) \mathrm{g} / \mathrm{cm}^{2}$ for $\mathrm{D}_{2}$, corrected for a $3 \% \mathrm{HD}$ admixture in $\mathrm{D}_{2}$. They were checked by continuously monitoring the vapour pressure of the target liquid. The small multi-wire proportional chamber $\mathrm{P} 0 \mathrm{~B}$ (see fig. 3), designed to operate at high intensities, was situated between the two targets to improve the resolution of the interaction vertex reconstruction; the chambers $\mathrm{P} 0 \mathrm{C}$ and PV1,2 downstream of the target served a similar purpose.

\subsection{THE SPECTROMETER}

The layout of the NMC spectrometer is presented in fig. 3. The incident muon momenta were measured in the beam momentum station and the beam halo was detected by a number of veto counters $\mathrm{V}$. The beam hodoscopes BHA and BHB determined the position of each incident muon to within $0.8 \mathrm{~mm}$ and its direction with a precision of $0.15 \mathrm{mrad}$. Neither the beam momentum station nor the beam hodoscopes were used in the electronic trigger.

The forward spectrometer magnet (FSM) with an aperture of $2 \times 1 \mathrm{~m}^{2}$ and a length of $4.3 \mathrm{~m}$ had a maximum field integral of $5.2 \mathrm{~T} \cdot \mathrm{m}$ which corresponds to a bending angle of $5.6 \mathrm{mrad}$ for $280 \mathrm{GeV}$ muons. Charged particles were tracked in a number of proportional and drift chambers. The proportional chambers P0B, P0C and PV1,2 placed before the FSM were used to determine the scattering angle. The chambers P0D and P1,2,3 in the FSM served for tracking particles in the magnetic field. The bending angle, and hence the particle momentum, was determined by tracking through the drift chambers $\mathrm{W} 1,2, \mathrm{~W} 4,5$ and the proportional chambers $\mathbf{P} 4,5$. All the large chambers had dead regions through which the beam passed. The small proportional chambers P0E and P0A covered the beam region. Muons were identified by tracks in the chambers W6,7 which were positioned behind a $2 \mathrm{~m}$ thick iron absorber. The FSM field map was calibrated by comparing 


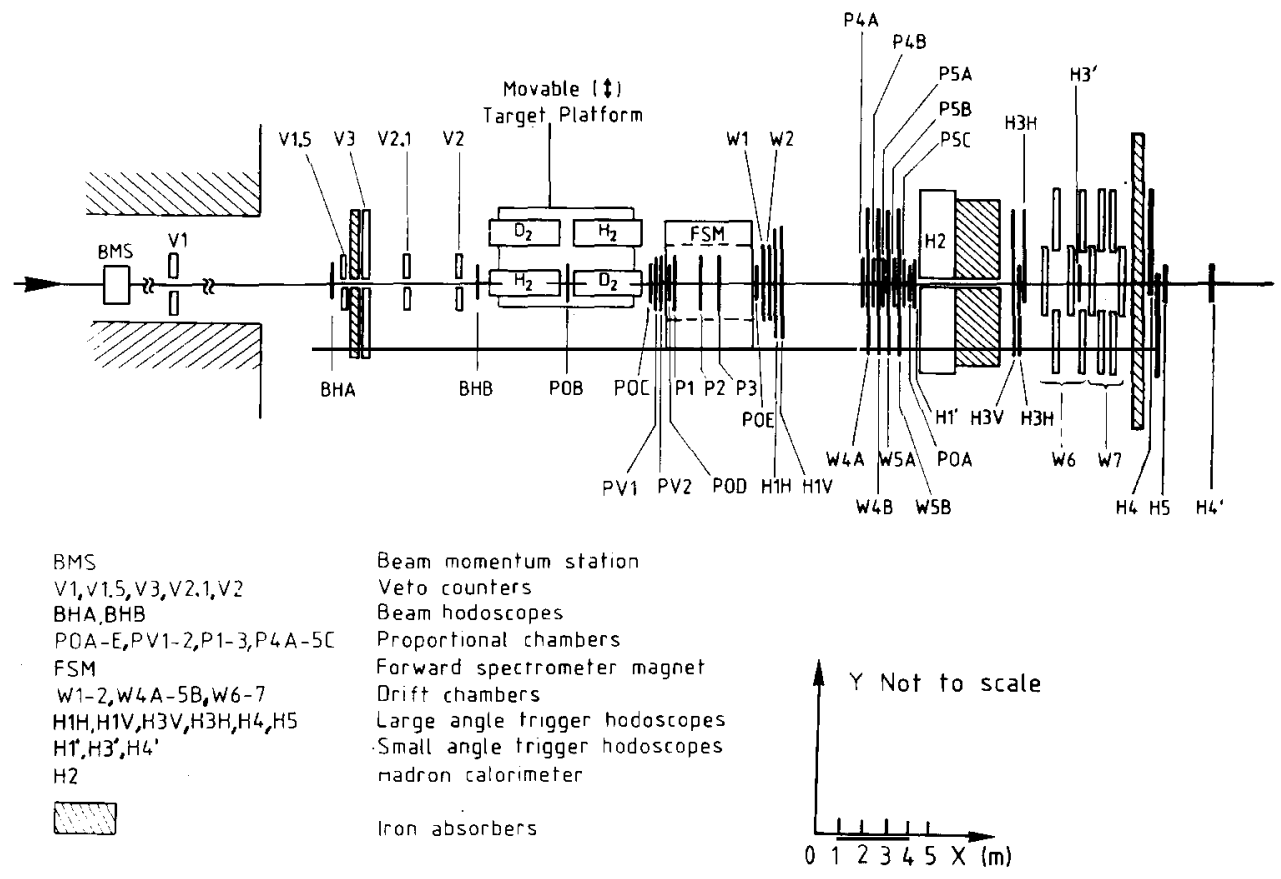

Fig. 3. The spectrometer of the New Muon Collaboration. The beam calibration spectrometer is not shown.

the observed $\mathrm{J} / \psi$ and $\mathrm{K}^{0}$ masses with their known values [14]. The estimated uncertainty on this calibration is $0.2 \%$.

\subsection{THE MUON TRIGGERS}

There were two triggers for scattered muons. Trigger T1 was sensitive to muons scattered at angles larger than $10 \mathrm{mrad}$ and the small angle trigger $\mathrm{T} 2$ selected muons at angles between 3 and $15 \mathrm{mrad}$. The triggers were formed using fast coincidence matrices [13] which required combinations of strips from the hodoscopes $\mathrm{H} 1, \mathrm{H} 3$ and $\mathrm{H} 4$ for $\mathrm{T} 1$ ( $\mathrm{H}^{\prime}, \mathrm{H}^{\prime}$ and $\mathrm{H}^{\prime}{ }^{\prime}$ for $\left.\mathrm{T} 2\right)$ such that the triggering particle was required to come from the target region. Combinations of strips which were mainly populated by radiative events were inhibited. The hodoscopes $\mathrm{H} 3$ and $\mathrm{H} 4$, and $\mathrm{H}^{\prime}$ and $\mathrm{H}^{\prime}$, were placed behind the iron absorber to remove hadrons from the trigger. A second $40 \mathrm{~cm}$ thick iron absorber was placed in front of $\mathrm{H} 4$ and $\mathrm{H}^{\prime}$ ' to shield these hodoscopes from electromagnetic showers created in the beam aperture through the hadron calorimeter and the first absorber wall. Beam halo was removed from the trigger by anticoincidence with the veto counters, which required that the incoming muon was within $3 \mathrm{~cm}$ of the beam axis at the position of V2. The total trigger rates were a few hundred per $2 \mathrm{~s}$ beam spill. 


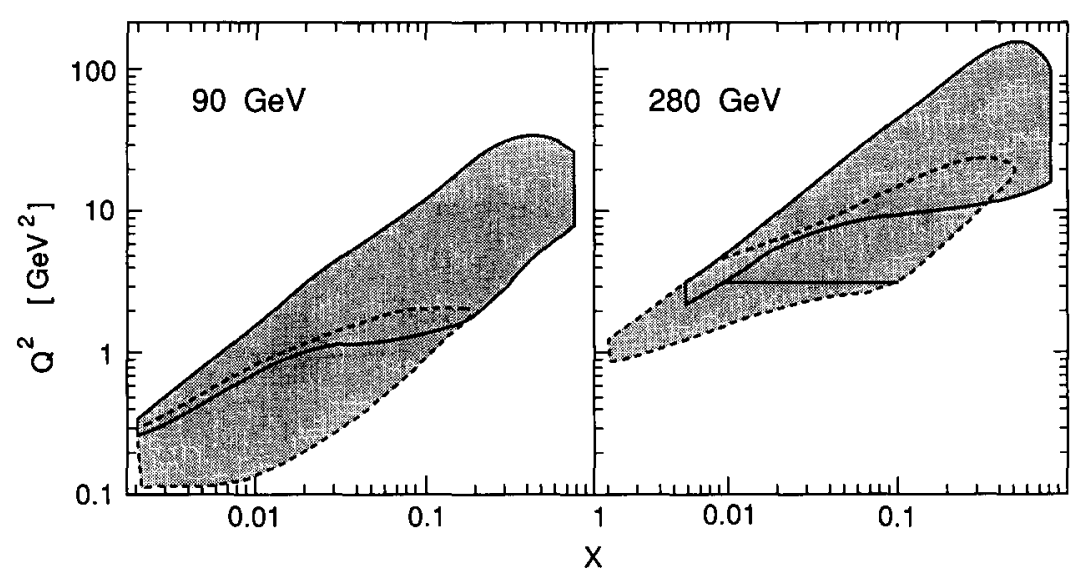

Fig. 4. The kinematic coverage in $x$ and $Q^{2}$ for triggers $T 1$ (full curves) and T2 (dashed curves) at 90 and $280 \mathrm{GeV}$ incident energies.

The kinematic ranges covered by $\mathrm{T} 1$ and $\mathrm{T} 2$ are given in fig. 4 for 90 and 280 GeV. Trigger T2 emphasises the small $x$ and $Q^{2}$ region. There is an appreciable overlap between the $\mathrm{T} 2$ data at $280 \mathrm{GeV}$ and those of $\mathrm{T} 1$ at $90 \mathrm{GeV}$, which enables a consistency check between results obtained from the two triggers.

\subsection{THE BEAM CALIBRATION SPECTROMETER}

Structure functions and their ratios are sensitive to uncertainties in the incident and scattered muon momenta. To determine the incident momentum with good precision a beam calibration spectrometer was installed behind the muon spectrometer. This spectrometer consisted of a $6 \mathrm{~m}$ long dipole magnet with a $14 \mathrm{~cm}$ gap providing a bend of $13 \mathrm{mrad}$ for $280 \mathrm{GeV}$ muons at a maximum field of $2 \mathrm{~T}$. The muons were detected in multiwire proportional chambers placed at the entry and exit points of the magnet and in a chamber located $35 \mathrm{~m}$ downstream. To obtain a sufficiently long lever arm to precisely determine the incoming muon track, the upstream chambers P0E, P0A and P4,5 were used. With this beam calibration spectrometer the beam momentum station was calibrated in dedicated runs to precisions of $0.2 \%(280 \mathrm{GeV})$ and $0.4 \%(90 \mathrm{GeV})$.

\section{The analysis}

\subsection{THE EVENT RECONSTRUCTION CHAIN}

The event reconstruction from the raw data tapes was performed in several steps. The most important were alignment and calibration of the apparatus, initial pattern recognition and track finding followed by track and vertex fitting. 
Alignment data were taken for each SPS period separately with the FSM magnet switched off using dedicated beam and beam halo triggers. From the alignment data accurate relative lateral positions of all detectors as well as drift chamber calibration constants were calculated.

The event reconstruction started with the incoming beam tracks. They were reconstructed in the beam hodoscopes and matched to the information from the beam momentum station. For events containing at least one good incident beam particle, the analysis program proceeded to find the scattered muon. A particle was assumed to be a muon if it was detected behind the hadron absorber. Therefore track segments were first looked for in the W6,7 chambers and extrapolated backwards through the absorber. Then a search was made for corresponding lines in $\mathrm{W} 4,5 / \mathrm{P} 4,5, \mathrm{~W} 1,2 / \mathrm{P} 0 \mathrm{E}$, in the magnet chambers $\mathrm{P} 0 \mathrm{D}, \mathrm{P} 1,2,3$ and upstream of the FSM in PV1,2/P0C and P0B. If none of the original muon candidates was successfully tracked up to the target, the event was rejected.

After this stage track segments were fitted together and the trigger conditions were checked for each muon. Then the incoming and scattered muon tracks were fitted to a vertex. The percentage of events which were fully reconstructed was $70 \%$ of the raw data sample at $90 \mathrm{GeV}$ and $20-25 \%$ at $280 \mathrm{GeV}$. These events were written to the data summary tapes and used in the subsequent analysis.

We do not describe the analysis of the hadrons in the data, which was only used here to reconstruct the $\mathrm{K}^{0}$ 's for the calibration of the FSM.

\subsection{EVENT SELECTION}

The final event sample was obtained from the reconstructed events by applying the kinematic cuts listed in table 1 . The total number of events used in the analysis after cuts was about 1.8 million for $90 \mathrm{GeV}$ (for $x>0.002$ and $Q^{2}>0.1 \mathrm{GeV}^{2}$ ) and 1.4 million for $280 \mathrm{GeV}$ (for $x>0.002$ and $Q^{2}>1.0 \mathrm{GeV}^{2}$ ).

The minimum scattered muon momentum cut removes most of the contamination from muons originating from hadron decays. The $\nu$ cut is made to avoid the kinematic region where $\nu$ is poorly determined (see also next section). The

TABLE 1

The kinematic cuts applied to the NMC data; the variables are defined in the laboratory frame

\begin{tabular}{llccc}
\hline \multicolumn{1}{c}{ Variable } & & $\begin{array}{c}90 \mathrm{GeV} \\
\text { data }\end{array}$ & $\begin{array}{c}280 \mathrm{GeV} \\
\text { data }\end{array}$ \\
\hline$y_{\max }$ & scaling variable $(\nu / E)$ & & 0.9 & 0.9 \\
$p_{\mu_{\min }}^{\prime}$ & scattered muon momentum & & $15 \mathrm{GeV}$ & $40 \mathrm{GeV}$ \\
$\theta_{\min }$ & muon scattering angle & $(\mathrm{T} 1)$ & $13 \mathrm{mrad}$ & $10 \mathrm{mrad}$ \\
& & $(\mathrm{T} 2)$ & $3 \mathrm{mrad}$ & $5 \mathrm{mrad}$ \\
$\nu_{\min }$ & energy transfer & $(\mathrm{T} 1)$ & $5 \mathrm{GeV}$ & $10 \mathrm{GeV}$ \\
& & $(\mathrm{T} 2)$ & $5 \mathrm{GeV}$ & $15 \mathrm{GeV}$ \\
\hline
\end{tabular}


maximum $y$ cut rejects the region with large radiative corrections. The $\left(x, Q^{2}\right)$ plane covered by the final data sample is shown in fig. 4 .

\subsection{CONSISTENCY AND SYSTEMATIC CHECKS}

As was pointed out in sect. 2, the method of extracting $F_{2}^{\mathrm{n}} / F_{2}^{\mathrm{p}}$ from the data ensures small systematic uncertainties provided that flux and acceptance cancel in the calculation of the ratio. In deriving eq. (3) use was made of the fact that the ratio of integrated beam fluxes incident upon the two target sets is not dependent on kinematic variables. Furthermore it was assumed that the detector acceptance was not strongly time dependent. This can be verified by monitoring the kinematic dependence of the flux ratio and the time dependence of the acceptance ratio, calculated from

$$
\frac{\Phi_{1}}{\Phi_{2}}=\sqrt{\frac{N_{\mathrm{d}}^{\mathrm{up}} N_{\mathrm{p}}^{\mathrm{dn}}}{N_{\mathrm{p}}^{\mathrm{up}} N_{\mathrm{d}}^{\mathrm{dn}}}}, \quad \frac{A^{\mathrm{up}}}{A^{\mathrm{dn}}}=\sqrt{\frac{N_{\mathrm{d}}^{\mathrm{up}} N_{\mathrm{p}}^{\mathrm{up}}}{N_{\mathrm{d}}^{\mathrm{dn}} N_{\mathrm{p}}^{\mathrm{dn}}}}
$$

for consecutive exposures of targets sets 1 and 2 .

The acceptance as introduced in eq. (2) includes the geometrical acceptance of the spectrometer, detector efficiencies and losses of the scattered muon due to the reconstruction algorithm and effects of high multiplicity in the chambers (background). Deviations of the acceptance ratio from the average by more than four standard deviations were due to known experimental problems and these data were removed from the analysis. No significant time dependence of the acceptance ratio was observed, as shown in fig. 5 for one period of data taking. The cross

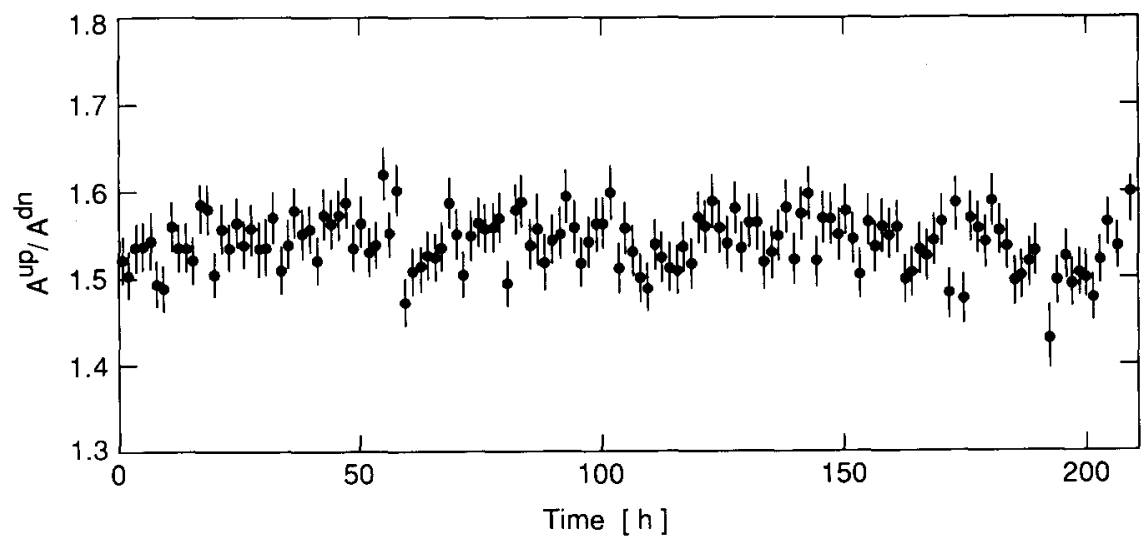

Fig. 5. The time dependence of the ratio of acceptances for the upstream and downstream targets for one period of data taking. 


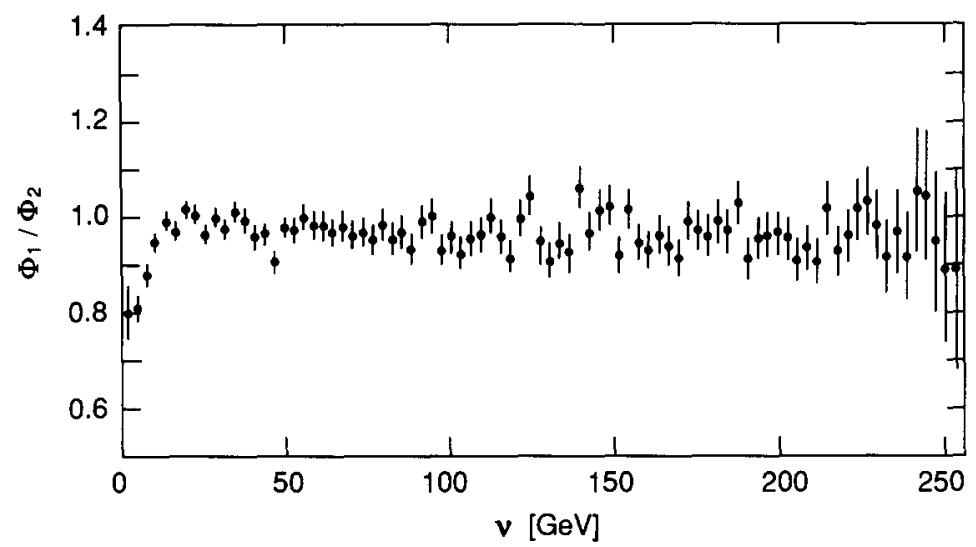

Fig. 6. The flux ratio $\Phi_{1} / \Phi_{2}$ for trigger $\mathrm{T} 2$ data at $280 \mathrm{GeV}$ incident energy as a function of the kinematic variable $\nu$ for one period of data taking before cuts were being applied.

section ratio was calculated separately for each such period and the results were merged.

The assumption of equal acceptances for both upstream and for both downstream targets was checked using the flux ratio defined by eq. (5); this ratio should not depend on any variable characterising the event. If necessary, cuts were applied in order to remove events from the edges of the kinematic regions where the flux ratio was no longer constant. For instance, the flux ratio for T2 data taken with $280 \mathrm{GeV}$ incident muon energy is shown as a function of $\nu$ in fig. 6. At low $\nu$, where a beam muon might be mistaken for a scattered muon, this ratio decreases and events with $\nu$ smaller than $15 \mathrm{GeV}$ were therefore removed. After applying the cuts given in table 1 , all significant kinematic dependences of the flux ratio were eliminated.

The acceptance might depend on the target material due to multiple scattering in hydrogen and deuterium and to background effects. Multiple scattering is similar in $\mathrm{H}_{2}$ and $\mathrm{D}_{2}$ and therefore its effect should cancel in the ratio. From a full Monte Carlo simulation of the experiment the main source of reconstruction losses due to background was found to be high multiplicity leading to dead times in the drift chambers. Although background effects in some parts of the detector seemed to be significant and also depended on the kinematics as well as on the incident energy, the reconstruction losses were found to be the same for the hydrogen and deuterium data. Their effects should therefore cancel in the ratio. Indeed, if one excludes from the analysis events possibly affected by background, the resulting structure function ratio $F_{2}^{\mathrm{n}} / F_{2}^{\mathrm{p}}$ is consistent with the one obtained from the full data sample.

As an additional check $F_{2}^{\mathrm{n}} / F_{2}^{\mathrm{p}}$ was calculated for the upstream and downstream targets separately, using the measured beam flux to normalise the data. No significant difference between these ratios was observed. 


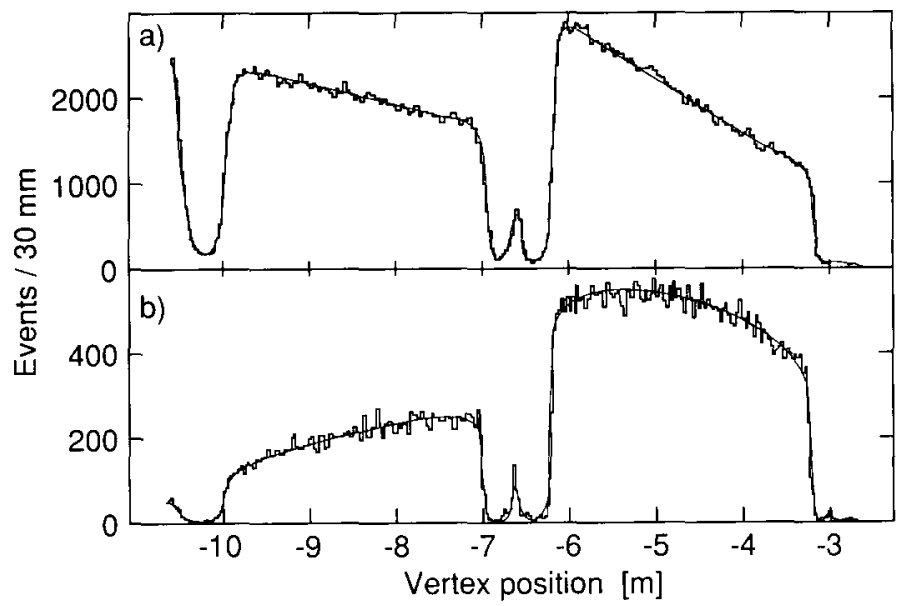

Fig. 7. Distribution of reconstructed longitudinal vertex positions in the upstream $\left(\mathrm{H}_{2}\right)$ and downstream ( $\mathrm{D}_{2}$ ) targets for scattering angles $\theta=13-20 \mathrm{mrad}$ (a) and $\theta>40 \mathrm{mrad}$ (b). The small peaks in the middle are caused by scattering in the chamber $\mathrm{P} 0 \mathrm{~B}$ and the rise near position $x=-10.5 \mathrm{~m}$ is due to scattering in the beam hodoscope BHB. The curves are fits to the data.

\subsection{CORRECTIONS AND SYSTEMATIC UNCERTAINTIES}

The finite resolution of the spectrometer leads to an uncertainty in the position of the interaction vertex. Consequently events might be wrongly associated to a target material or might fall outside of the target region. To estimate the number of such events, the vertex distributions were fitted. As the vertex resolution depends strongly on the scattering angle $\theta$ these fits were performed in $\theta$ intervals. In fig. 7 the vertex distributions are shown for the lowest and highest $\theta$ bin together with the fitted curves. These were used to determine the tails of the vertex distributions. Correction factors which accounted for wrong target association were calculated for the average $\theta$ in each $\left(x, Q^{2}\right)$ bin. The size of this correction varied between $1.2 \%$ and $0.2 \%$ and its error was assumed to be half of the value.

In order to correct for the effects of kinematic smearing in $x$ and $Q^{2}$, a Monte Carlo simulation of the experiment with reconstruction of the generated muon tracks was performed. These corrections were usually below $1 \%$ and the errors always negligible.

All corrections mentioned above were calculated for each period of data taking separately. In addition the effect of the 3\% HD admixture in the deuterium was taken into account for each kinematic bin separately. The final results were obtained by merging all corrected data sets.

The results on the ratio $F_{2}^{\mathrm{n}} / F_{2}^{\mathrm{p}}$ at high $x$ are sensitive to uncertainties in the incoming and scattered muon momenta (see subsects. 3.2 and 3.5). An analytical method was used [15] to calculate the changes in $F_{2}^{\mathrm{n}} / F_{2}^{\mathrm{p}}$ due to these uncertain- 
ties. These changes were combined in quadrature to give the corresponding systematic error.

The uncertainties in the hydrogen and deuterium densities and target lengths lead to a normalisation error which is smaller than $0.15 \%$.

\subsection{RADIATIVE CORRECTIONS}

The structure function ratio $F_{2}^{\mathrm{n}} / F_{2}^{\mathrm{p}}$ was obtained from the measured total cross section ratio for hydrogen and deuterium by applying radiative corrections, calculated with the method of Tsai and Mo and Tsai [16]. For the calculation of these corrections the structure functions $F_{2}^{\mathrm{p}}$ and $F_{2}^{\mathrm{d}}$ are needed. Therefore the extraction of $F_{2}^{\mathrm{n}} / F_{2}^{\mathrm{p}}$ was performed using an iterative procedure. In this procedure, $F_{2}^{\mathrm{d}}$ was fixed to a function from a fit to previous experimental data, whereas $F_{2}^{\mathrm{p}}$ was obtained by combining this $F_{2}^{\mathrm{d}}$ with the presently measured ratio $F_{2}^{\mathrm{n}} / F_{2}^{\mathrm{p}}$. Outside the measured kinematic range we used $F_{2}^{p}$ from a fit to results of other experiments. The iteration was stopped (usually after three steps) when the change in $F_{2}^{\mathrm{n}} / F_{2}^{\mathrm{p}}$ at any point in $x$ and $Q^{2}$ was less than $0.2 \%$.

The calculation of radiative corrections includes the exact treatment of the elastic and quasi-elastic radiative tails, an approximate treatment of the inelastic tail, vacuum polarisation loops $\left(\mathrm{e}^{+} \mathrm{e}^{-}\right.$and $\left.\mu^{+} \mu^{-}\right)$and a partial treatment of $\alpha^{4}$ contributions to the lepton current.

In the determination of the inelastic tails one needs the structure function $F_{2}^{\mathrm{d}}$ and $R$. For the function $R$ the parametrisation of ref. [9] was taken for all $x$ and $Q^{2}>0.35 \mathrm{GeV}^{2}$. For smaller $Q^{2}$ the value of $R$ was assumed to be constant and taken equal to the value at $Q^{2}=0.35 \mathrm{GeV}^{2}$.

The structure function $F_{2}^{\mathrm{d}}$ was obtained using the following procedure. In the resonance region $F_{2}^{\text {d }}$ was fitted to the data from SLAC [17] taking only the $\Delta(1232)$ resonance into account. Outside the resonance region a QCD based parametrisation was used to describe the data of SLAC [9], BCDMS [18], EMCNA28 [19] and CHIO [20]. The relative normalisation between the various data sets was not adjusted and only statistical errors were used as weights. A detailed description of the parametrisation and the values obtained for the parameters can be found in appendix $A$.

For the evaluation of the proton elastic tail, the nucleon form factors from Höhler's parametrisation [21] were used. They were also used to calculate the quasi-elastic tail for scattering on the deuteron. The reduction of the deuteron elastic cross section per nucleon with respect to that of the free nucleon was calculated using the model of Bernabeu [22]. For the estimation of the coherent tail for the deuteron we used the form factor from a fit to all available data by Locher and Švarc [23].

An alternative calculation of the radiative corrections [24] includes electro-weak interference and a more complete treatment of higher-order processes. The 
differences between the results of the two methods are at most a few percent [25] and are negligible for $F_{2}^{\mathrm{n}} / F_{2}^{\mathrm{p}}$.

At the same $x$ and $Q^{2}$ the data obtained at the two energies have different $y$ and therefore substantially different radiative corrections at low $x$. No differences between the two data sets were observed which cannot be accommodated within a small $Q^{2}$ dependence of $F_{2}^{\mathrm{n}} / F_{2}^{\mathrm{p}}$. As an additional check of the radiative correction and iteration procedure the analysis was repeated keeping $F_{2}^{\mathrm{p}}$ fixed while modifying $F_{2}^{\mathrm{d}}$ [26]. The function $F_{2}^{\mathrm{p}}$ was obtained from a fit similar to that described for $F_{2}^{\mathrm{d}}$. The resulting ratio $F_{2}^{\mathrm{n}} / F_{2}^{\mathrm{p}}$ agreed with the previous one within the systematic errors due to radiative corrections.

The most important contributions to the systematic error on the ratio $F_{2}^{\mathrm{n}} / F_{2}^{\mathrm{p}}$ due to the applied radiative corrections stem from the uncertainties in $R$ and $F_{2}^{\mathrm{d}}$. The systematic error was estimated as follows. A lower limit for $F_{2}^{\mathrm{d}}$ was obtained from a fit in which all data were simultaneously lowered by their quoted normalisation errors, which were also included in the weights. Similarly an upper limit was obtained by raising all data by their normalisation uncertainty. The differences between these limits and the standard fit were taken as an estimate of the systematic uncertainty on $F_{2}^{\mathrm{d}}$. The systematic error on $R$ was taken from ref. [9] for $Q^{2} \geqslant 0.35 \mathrm{GeV}^{2}$. For lower values of $Q^{2}$ the uncertainty in $R$ was assumed to be its value. As to the other inputs, i.e. the proton form factor, the deuteron form factor and the quasi-elastic treatment, alternative sets due to Atwood [27], Stein et al. [5] and Arenhövel [28], respectively, were used to obtain estimates of their uncertainties. After calculating the influence of every contributing uncertainty on $F_{2}^{\mathrm{n}} / F_{2}^{\mathrm{p}}$ separately, two sets of inputs were selected, one giving maximum, the other giving minimum values of $F_{2}^{\mathrm{n}} / F_{2}^{\mathrm{p}}$. In each case $R$ was taken to be the same for the proton and deuteron. The above described iterative procedure was repeated with each set. The average difference between the original value of $F_{2}^{\mathrm{n}} / F_{2}^{\mathrm{p}}$ and the other two was taken as the total systematic error due to radiative corrections. Contributions to the systematic error from the numerical precision of the integrations and the influence of the infrared cut-off were estimated to be smaller than $0.6 \%$.

\section{The results}

\subsection{THE $x$-DEPENDENCE}

The results for $F_{2}^{\mathrm{n}} / F_{2}^{\mathrm{p}}$ at the centre of each $x$ bin and averaged over $Q^{2}$ are presented in fig. 8 and listed in table 2 for 90 and $280 \mathrm{GeV}$ separately. The bands in the figure show the size of the systematic errors. The results for the two energies were combined and are listed in table 3 together with the separate contributions to the total systematic error. The main contribution to this error at small $x$ comes 


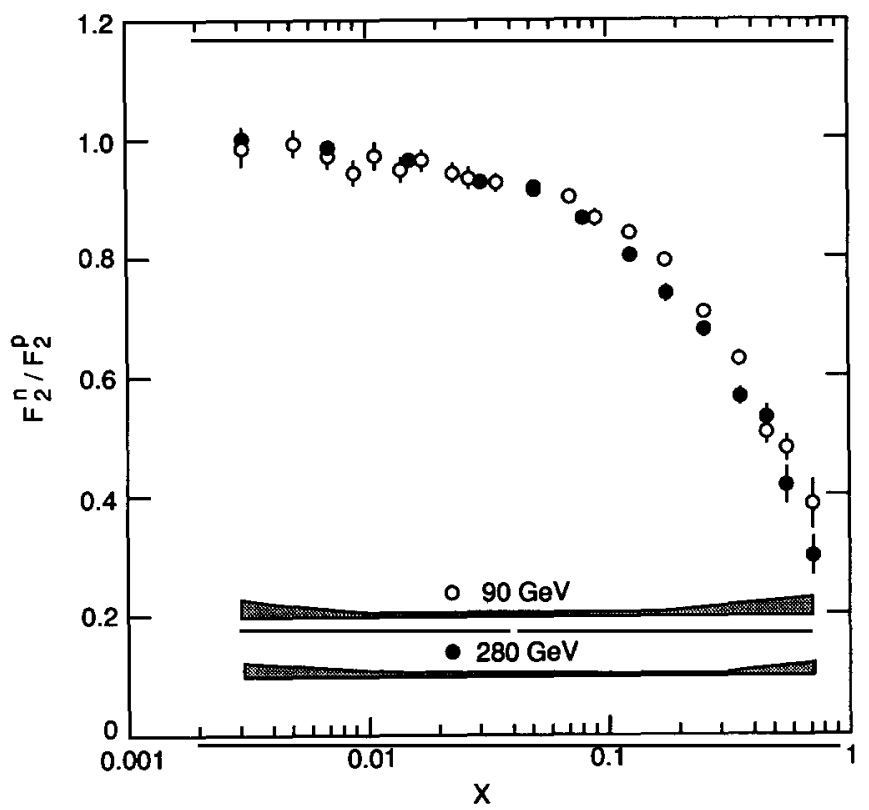

Fig. 8. The structure function ratio $F_{2}^{\mathrm{n}} / F_{2}^{\mathrm{p}}$ averaged over $Q^{2}$ as a function of $x$ for the 90 and 280 $\mathrm{GeV}$ data. The statistical errors are represented by the error bars. The bands at the bottom indicate the systematic uncertainties.

from radiative corrections whereas at large $x$ the uncertainties in the incoming and outgoing muon momenta dominate. No corrections to $F_{2}^{\mathrm{n}} / F_{2}^{\mathrm{p}}$ for nuclear effects have been applied; those for Fermi motion are small below $x=0.6$ [4]. The data presented here include those previously published $[10,11]$ and extend to lower $x$ and $Q^{2}$; in particular the small-angle trigger T2 data for $90 \mathrm{GeV}$ were added.

It has been pointed out [10] that the ratio can be used to constrain parametrisations of parton distributions [29] notably in the region $x=0.01-0.1$, where valence and sea partons give comparable contributions. The data constrain the quark $d / u$ ratio which is needed to predict hard scattering cross section ratios in ep, pp and $\mathrm{p} \overline{\mathrm{p}}$ collisions.

The NMC results on $F_{2}^{\mathrm{n}} / F_{2}^{\mathrm{p}}$, together with the data on $F_{2}^{\mathrm{d}}$ from other experiments, have also been used to calculate the value of the Gottfried sum at $Q^{2}=4 \mathrm{GeV}^{2}[11]$. The present extension of the kinematic region to $x=0.002$ does not alter the value of the sum significantly.

At the lowest measured value $x=0.003$ where $\left\langle Q^{2}\right\rangle=0.62 \mathrm{GeV}^{2}$ we find $F_{2}^{\mathrm{n}} / F_{2}^{\mathrm{p}}=0.990 \pm 0.016 \pm 0.026$, consistent with unity. There is no evidence for sizeable shadowing in deuterium which would manifest itself as a suppression of the ratio at low $x$. However the data can not exclude a 2-3\% effect from shadowing at $x=0.002$ as predicted in ref. [30]. 
TABLE 2

The ratio $F_{2}^{\mathrm{n}} / F_{2}^{\mathrm{p}}$ averaged over $Q^{2}$ for the 90 and $280 \mathrm{GeV}$ data

\begin{tabular}{|c|c|c|c|c|c|c|c|c|c|}
\hline \multicolumn{5}{|c|}{ Mean energy $89 \mathrm{GeV}$} & \multicolumn{5}{|c|}{ Mean energy $274 \mathrm{GeV}$} \\
\hline \multirow[t]{2}{*}{$x$} & \multirow[t]{2}{*}{$\left\langle Q^{2}\right\rangle$} & \multirow[t]{2}{*}{$F_{2}^{\mathrm{n}} / F_{2}^{\mathrm{p}}$} & \multicolumn{2}{|c|}{ Error } & \multirow[t]{2}{*}{$x$} & \multirow[t]{2}{*}{$\left\langle Q^{2}\right\rangle$} & \multirow[t]{2}{*}{$F_{2}^{\mathrm{n}} / F_{2}^{\mathrm{p}}$} & \multicolumn{2}{|c|}{ Error } \\
\hline & & & stat. & syst. & & & & stat. & syst. \\
\hline 0.003 & 0.4 & 0.986 & 0.027 & 0.028 & 0.003 & 1.0 & 1.001 & 0.025 & 0.022 \\
\hline 0.005 & 0.5 & 0.992 & 0.023 & 0.015 & & & & & \\
\hline 0.007 & 0.7 & 0.971 & 0.022 & 0.010 & 0.007 & 2.5 & 0.981 & 0.012 & 0.012 \\
\hline 0.009 & 0.9 & 0.943 & 0.023 & 0.007 & & & & & \\
\hline 0.011 & 1.0 & 0.971 & 0.024 & 0.007 & & & & & \\
\hline 0.014 & 1.2 & 0.947 & 0.020 & 0.006 & 0.015 & 4.5 & 0.960 & 0.010 & 0.007 \\
\hline 0.017 & 1.4 & 0.964 & 0.017 & 0.006 & & & & & \\
\hline 0.023 & 1.7 & 0.940 & 0.017 & 0.006 & & & & & \\
\hline 0.027 & 1.9 & 0.932 & 0.018 & 0.006 & 0.030 & 7.6 & 0.926 & 0.009 & 0.005 \\
\hline 0.035 & 2.2 & 0.925 & 0.014 & 0.006 & & & & & \\
\hline 0.050 & 2.6 & 0.918 & 0.011 & 0.005 & 0.050 & 11.0 & 0.913 & 0.011 & 0.004 \\
\hline 0.070 & 3.1 & 0.901 & 0.012 & 0.005 & & & & & \\
\hline 0.090 & 3.5 & 0.864 & 0.013 & 0.005 & 0.080 & 14.4 & 0.863 & 0.009 & 0.003 \\
\hline 0.125 & 3.9 & 0.840 & 0.009 & 0.005 & 0.125 & 20.0 & 0.803 & 0.011 & 0.003 \\
\hline 0.175 & 4.6 & 0.794 & 0.010 & 0.006 & 0.175 & 25.5 & 0.739 & 0.013 & 0.004 \\
\hline 0.250 & 5.6 & 0.710 & 0.009 & 0.010 & 0.250 & 30.8 & 0.679 & 0.012 & 0.004 \\
\hline 0.350 & 7.1 & 0.629 & 0.014 & 0.015 & 0.350 & 36.3 & 0.564 & 0.016 & 0.006 \\
\hline 0.450 & 8.2 & 0.504 & 0.021 & 0.022 & 0.450 & 37.1 & 0.530 & 0.022 & 0.008 \\
\hline 0.550 & 9.4 & 0.479 & 0.033 & 0.026 & 0.550 & 38.7 & 0.414 & 0.030 & 0.012 \\
\hline 0.700 & 10.8 & 0.383 & 0.042 & 0.017 & 0.700 & 36.3 & 0.295 & 0.032 & 0.017 \\
\hline
\end{tabular}

\subsection{THE $Q^{2}$ DEPENDENCE}

At a given $x$ the average $Q^{2}$ is different for data taken at different energies. Thus the small differences seen in the $x$ dependence of $F_{2}^{\mathrm{n}} / F_{2}^{\mathrm{p}}$ obtained at 90 and

TABLE 3

The ratio $F_{2}^{\mathrm{n}} / F_{2}^{\mathrm{p}}$ averaged over $Q^{2}$ for the merged 90 and $280 \mathrm{GeV}$ data

\begin{tabular}{cccccccc}
\hline$x$ & $\left\langle Q^{2}\right\rangle$ & $F_{2}^{\mathrm{n}} / F_{2}^{\mathrm{p}}$ & $\begin{array}{c}\text { Statistical } \\
\text { error }\end{array}$ & & \multicolumn{3}{c}{ Systematic error } \\
\cline { 5 - 7 } & & & & rad. corr. & momentum & other & total \\
\hline 0.003 & 0.6 & 0.990 & 0.016 & 0.025 & 0.000 & 0.005 & 0.026 \\
0.007 & 1.4 & 0.971 & 0.008 & 0.010 & 0.000 & 0.005 & 0.011 \\
0.015 & 2.6 & 0.959 & 0.007 & 0.005 & 0.000 & 0.005 & 0.006 \\
0.030 & 4.2 & 0.927 & 0.006 & 0.002 & 0.000 & 0.005 & 0.005 \\
0.050 & 5.9 & 0.915 & 0.007 & 0.001 & 0.000 & 0.005 & 0.005 \\
0.080 & 7.7 & 0.874 & 0.006 & 0.001 & 0.001 & 0.004 & 0.004 \\
0.125 & 10.0 & 0.825 & 0.006 & 0.000 & 0.002 & 0.004 & 0.004 \\
0.175 & 12.3 & 0.774 & 0.008 & 0.000 & 0.004 & 0.003 & 0.005 \\
0.250 & 15.2 & 0.700 & 0.007 & 0.000 & 0.006 & 0.003 & 0.007 \\
0.350 & 20.2 & 0.588 & 0.011 & 0.000 & 0.009 & 0.002 & 0.009 \\
0.450 & 22.3 & 0.513 & 0.015 & 0.000 & 0.012 & 0.002 & 0.013 \\
0.550 & 26.0 & 0.431 & 0.022 & 0.000 & 0.013 & 0.002 & 0.013 \\
0.700 & 27.6 & 0.317 & 0.026 & 0.000 & 0.009 & 0.002 & 0.009 \\
\hline
\end{tabular}




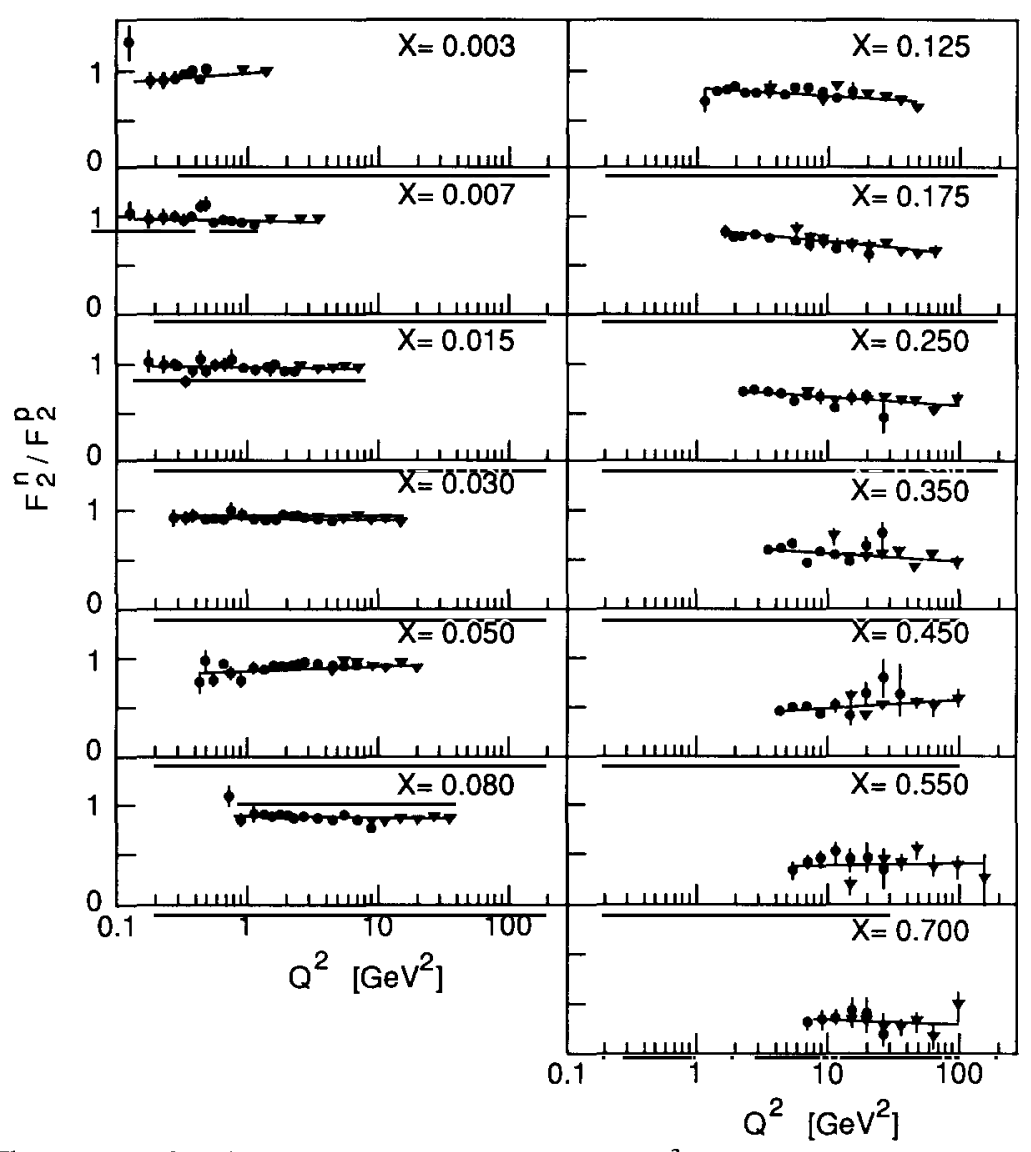

Fig. 9. The structure function ratio $F_{2}^{\mathrm{n}} / F_{2}^{\mathrm{p}}$ as a function of $Q^{2}$ for each $x$ bin. The $90 \mathrm{GeV}$ data are represented by circles, the $280 \mathrm{GeV}$ data by triangles. Only statistical errors are given. Fits of linear functions of $\ln Q^{2}$ to the data are also shown.

$280 \mathrm{GeV}$ (fig. 8) indicate a $Q^{2}$ dependence of the ratio. This dependence is presented in fig. 9 for the two data sets. In the overlap region the data from both incident energies are in good agreement and they were combined to give the results presented in table 4 .

The combined data cover the $Q^{2}$ range $0.1-190 \mathrm{GeV}^{2}$. For each $x$ bin the data were fitted with a linear function of $\ln Q^{2}$,

$$
F_{2}^{\mathrm{n}} / F_{2}^{\mathrm{p}}\left(x_{i}, Q^{2}\right)=a\left(x_{i}\right)+b\left(x_{i}\right) \ln Q^{2},
$$

also shown in fig. 9. In table 5 the results of these fits are compared to those without any $Q^{2}$ dependence (i.e. with $b\left(x_{i}\right)=0$ in eq. (6)). The fitted parameter $b$ and fit probabilities for each $x$ bin are given. The fits indicate significant negative slopes in the $x$ range $0.1-0.4$. At lower $x$ no $Q^{2}$ dependence is observed. 
TABLE 4

The ratio $F_{2}^{\mathrm{n}} / F_{2}^{\mathrm{p}}$ in bins of $x$ and $Q^{2}$ for the merged 90 and $280 \mathrm{GeV}$ data

\begin{tabular}{|c|c|c|c|c|c|c|c|}
\hline \multirow[t]{2}{*}{$Q^{2}$} & \multirow[t]{2}{*}{$F_{2}^{\mathrm{n}} / F_{2}^{\mathrm{p}}$} & \multicolumn{2}{|c|}{ Error } & \multirow[t]{2}{*}{$Q^{2}$} & \multirow[t]{2}{*}{$F_{2}^{\mathrm{n}} / F_{2}^{\mathrm{p}}$} & \multicolumn{2}{|c|}{ Error } \\
\hline & & stat. & syst. & & & stat. & syst. \\
\hline \multicolumn{4}{|c|}{$x=0.003$} & 2.25 & 0.917 & 0.036 & 0.006 \\
\hline 0.125 & 1.299 & 0.172 & 0.021 & 2.5 & 0.970 & 0.024 & 0.004 \\
\hline 0.175 & 0.898 & 0.092 & 0.008 & 3.5 & 0.947 & 0.025 & 0.005 \\
\hline 0.225 & 0.918 & 0.076 & 0.008 & 4.5 & 0.951 & 0.022 & 0.009 \\
\hline 0.275 & 0.929 & 0.081 & 0.026 & 5.5 & 0.981 & 0.025 & 0.010 \\
\hline 0.325 & 0.974 & 0.051 & 0.039 & 7.0 & 0.959 & 0.024 & 0.010 \\
\hline 0.375 & 1.016 & 0.045 & 0.038 & & & & \\
\hline 0.425 & 0.921 & 0.041 & 0.029 & \multicolumn{4}{|c|}{$x=0.030$} \\
\hline 0.475 & 1.040 & 0.052 & 0.029 & 0.275 & 0.937 & 0.081 & 0.028 \\
\hline 0.900 & 1.018 & 0.038 & 0.024 & 0.325 & 0.926 & 0.067 & 0.003 \\
\hline 1.375 & 0.992 & 0.034 & 0.020 & 0.375 & 0.971 & 0.057 & 0.097 \\
\hline & & & & 0.425 & 0.820 & 0.057 & 0.022 \\
\hline \multicolumn{4}{|c|}{$x=0.007$} & 0.475 & 0.909 & 0.064 & 0.031 \\
\hline 0.125 & 1.047 & 0.140 & 0.018 & 0.550 & 0.872 & 0.046 & 0.013 \\
\hline 0.175 & 0.985 & 0.095 & 0.018 & 0.650 & 0.872 & 0.052 & 0.062 \\
\hline 0.225 & 1.006 & 0.077 & 0.014 & 0.750 & 1.013 & 0.067 & 0.033 \\
\hline 0.275 & 1.012 & 0.063 & 0.012 & 0.900 & 0.973 & 0.062 & 0.025 \\
\hline 0.325 & 0.979 & 0.064 & 0.008 & 1.125 & 0.922 & 0.029 & 0.006 \\
\hline 0.375 & 1.015 & 0.062 & 0.004 & 1.375 & 0.921 & 0.021 & 0.007 \\
\hline 0.425 & 1.112 & 0.074 & 0.005 & 1.625 & 0.924 & 0.020 & 0.024 \\
\hline 0.475 & 1.128 & 0.084 & 0.009 & 1.875 & 0.976 & 0.022 & 0.019 \\
\hline 0.550 & 0.951 & 0.025 & 0.014 & 2.25 & 0.946 & 0.017 & 0.005 \\
\hline 0.650 & 0.984 & 0.025 & 0.016 & 2.50 & 0.942 & 0.029 & 0.012 \\
\hline 0.750 & 0.967 & 0.025 & 0.014 & 2.75 & 0.924 & 0.021 & 0.008 \\
\hline 0.900 & 0.947 & 0.020 & 0.010 & 3.5 & 0.915 & 0.017 & 0.012 \\
\hline 1.125 & 0.924 & 0.030 & 0.012 & 4.5 & 0.896 & 0.025 & 0.002 \\
\hline 1.5 & 0.977 & 0.021 & 0.010 & 5.5 & 0.918 & 0.037 & 0.024 \\
\hline 2.5 & 0.972 & 0.018 & 0.013 & 7.0 & 0.960 & 0.024 & 0.025 \\
\hline 3.5 & 0.986 & 0.025 & 0.014 & 9.0 & 0.912 & 0.020 & 0.005 \\
\hline \multirow{2}{*}{\multicolumn{4}{|c|}{$x=0.015$}} & 11.5 & 0.928 & 0.021 & 0.023 \\
\hline & & & & 15 & 0.887 & 0.034 & 0.016 \\
\hline 0.175 & 1.020 & 0.108 & 0.025 & & & & \\
\hline 0.225 & 0.999 & 0.087 & 0.024 & \multicolumn{4}{|c|}{$x=0.050$} \\
\hline 0.275 & 0.998 & 0.072 & 0.015 & 0.425 & 0.762 & 0.124 & 0.021 \\
\hline 0.325 & 0.825 & 0.064 & 0.013 & 0.475 & 0.974 & 0.104 & 0.023 \\
\hline 0.375 & 0.933 & 0.064 & 0.009 & 0.550 & 0.777 & 0.055 & 0.021 \\
\hline 0.425 & 1.055 & 0.076 & 0.009 & 0.650 & 0.938 & 0.065 & 0.023 \\
\hline 0.475 & 0.930 & 0.070 & 0.009 & 0.750 & 0.848 & 0.071 & 0.022 \\
\hline 0.550 & 0.997 & 0.055 & 0.005 & 0.900 & 0.764 & 0.062 & 0.012 \\
\hline 0.650 & 1.003 & 0.065 & 0.006 & 1.125 & 0.912 & 0.051 & 0.009 \\
\hline 0.750 & 1.050 & 0.082 & 0.003 & 1.375 & 0.882 & 0.034 & 0.006 \\
\hline 0.900 & 0.962 & 0.027 & 0.006 & 1.625 & 0.930 & 0.030 & 0.006 \\
\hline 1.125 & 0.944 & 0.018 & 0.006 & 1.875 & 0.916 & 0.028 & 0.006 \\
\hline 1.375 & 0.973 & 0.019 & 0.007 & 2.25 & 0.926 & 0.021 & 0.005 \\
\hline 1.500 & 0.927 & 0.048 & 0.004 & 2.50 & 0.934 & 0.042 & 0.004 \\
\hline 1.625 & 0.995 & 0.024 & 0.006 & 2.75 & 0.967 & 0.025 & 0.004 \\
\hline 1.875 & 0.916 & 0.031 & 0.006 & 3.5 & 0.929 & 0.018 & 0.003 \\
\hline
\end{tabular}


The New Muon Collaboration / Deep inelastic muon scattering

TABle 4 (CONTINUED)

\begin{tabular}{|c|c|c|c|c|c|c|c|}
\hline \multirow[t]{2}{*}{$Q^{2}$} & \multirow[t]{2}{*}{$F_{2}^{\mathrm{n}} / F_{2}^{\mathrm{p}}$} & \multicolumn{2}{|c|}{ Error } & \multirow[t]{2}{*}{$Q^{2}$} & \multirow[t]{2}{*}{$F_{2}^{\mathrm{n}} / F_{2}^{\mathrm{p}}$} & \multicolumn{2}{|c|}{ Error } \\
\hline & & stat. & syst. & & & stat. & syst. \\
\hline 4.5 & 0.879 & 0.023 & 0.003 & 3.5 & 0.810 & 0.023 & 0.029 \\
\hline 5.5 & 0.930 & 0.031 & 0.003 & 4.5 & 0.748 & 0.027 & 0.021 \\
\hline 7.0 & 0.945 & 0.036 & 0.003 & 5.5 & 0.807 & 0.031 & 0.026 \\
\hline 9.0 & 0.911 & 0.044 & 0.006 & 7.0 & 0.760 & 0.027 & 0.002 \\
\hline 11.5 & 0.901 & 0.027 & 0.003 & 9.0 & 0.783 & 0.036 & 0.017 \\
\hline 15 & 0.958 & 0.024 & 0.003 & 11.5 & 0.736 & 0.037 & 0.009 \\
\hline \multirow[t]{3}{*}{20} & 0.905 & 0.028 & 0.004 & 15 & 0.762 & 0.034 & 0.026 \\
\hline & & & & 20 & 0.714 & 0.028 & 0.024 \\
\hline & \multicolumn{2}{|c|}{$x=0.080$} & & 27 & 0.765 & 0.031 & 0.036 \\
\hline 0.750 & 1.093 & 0.097 & 0.145 & 36 & 0.697 & 0.036 & 0.022 \\
\hline 0.900 & 0.846 & 0.059 & 0.018 & 48 & 0.655 & 0.044 & 0.021 \\
\hline 1.125 & 0.916 & 0.071 & 0.013 & 65 & 0.699 & 0.060 & 0.117 \\
\hline 1.375 & 0.900 & 0.035 & 0.010 & & & & \\
\hline 1.625 & 0.893 & 0.028 & 0.004 & \multicolumn{4}{|c|}{$x=0.250$} \\
\hline 1.875 & 0.911 & 0.026 & 0.023 & 2.25 & 0.742 & 0.033 & 0.015 \\
\hline 2.25 & 0.875 & 0.019 & 0.003 & 2.75 & 0.760 & 0.025 & 0.015 \\
\hline 2.50 & 0.877 & 0.047 & 0.010 & 3.5 & 0.748 & 0.020 & 0.012 \\
\hline 2.75 & 0.905 & 0.021 & 0.024 & 4.5 & 0.724 & 0.024 & 0.009 \\
\hline 3.5 & 0.864 & 0.015 & 0.008 & 5.5 & 0.660 & 0.029 & 0.007 \\
\hline 4.5 & 0.854 & 0.019 & 0.027 & 7.0 & 0.689 & 0.025 & 0.005 \\
\hline 5.5 & 0.913 & 0.025 & 0.035 & 9.0 & 0.687 & 0.031 & 0.004 \\
\hline 7.0 & 0.848 & 0.022 & 0.029 & 11.5 & 0.621 & 0.031 & 0.003 \\
\hline 9.0 & 0.796 & 0.032 & 0.013 & 15 & 0.698 & 0.031 & 0.004 \\
\hline 11.5 & 0.832 & 0.031 & 0.018 & 20 & 0.699 & 0.025 & 0.003 \\
\hline 15 & 0.879 & 0.023 & 0.011 & 27 & 0.690 & 0.026 & 0.002 \\
\hline 20 & 0.858 & 0.020 & 0.021 & 36 & 0.683 & 0.031 & 0.002 \\
\hline 27 & 0.903 & 0.027 & 0.017 & 48 & 0.666 & 0.037 & 0.002 \\
\hline \multirow[t]{3}{*}{36} & 0.873 & 0.048 & 0.174 & 65 & 0.568 & 0.044 & 0.001 \\
\hline & & & & 100 & 0.689 & 0.065 & 0.001 \\
\hline & \multicolumn{3}{|c|}{$x=0.125$} & & & & \\
\hline 1.125 & 0.741 & 0.111 & 0.007 & \multicolumn{4}{|c|}{$x=0.350$} \\
\hline 1.375 & 0.847 & 0.043 & 0.007 & 3.5 & 0.625 & 0.031 & 0.025 \\
\hline 1.625 & 0.853 & 0.033 & 0.026 & 4.5 & 0.650 & 0.033 & 0.020 \\
\hline 1.875 & 0.910 & 0.031 & 0.047 & 5.5 & 0.706 & 0.041 & 0.016 \\
\hline 2.25 & 0.831 & 0.021 & 0.004 & 7.0 & 0.503 & 0.032 & 0.012 \\
\hline 2.75 & 0.838 & 0.023 & 0.012 & 9.0 & 0.609 & 0.047 & 0.008 \\
\hline 3.5 & 0.824 & 0.018 & 0.016 & 11.5 & 0.633 & 0.043 & 0.006 \\
\hline 4.5 & 0.799 & 0.020 & 0.037 & 15 & 0.527 & 0.038 & 0.005 \\
\hline 5.5 & 0.879 & 0.026 & 0.007 & 20 & 0.580 & 0.033 & 0.003 \\
\hline 7.0 & 0.871 & 0.024 & 0.039 & 27 & 0.600 & 0.034 & 0.003 \\
\hline 9.0 & 0.799 & 0.032 & 0.018 & 36 & 0.616 & 0.040 & 0.002 \\
\hline 11.5 & 0.842 & 0.034 & 0.022 & 48 & 0.459 & 0.042 & 0.002 \\
\hline 15 & 0.806 & 0.028 & 0.004 & 65 & 0.589 & 0.058 & 0.001 \\
\hline 20 & 0.822 & 0.024 & 0.012 & 100 & 0.510 & 0.060 & 0.001 \\
\hline 27 & 0.799 & 0.025 & 0.024 & & & & \\
\hline 36 & 0.754 & 0.032 & 0.041 & \multicolumn{4}{|c|}{$x=0.450$} \\
\hline \multirow[t]{3}{*}{48} & 0.674 & 0.043 & 0.072 & 4.5 & 0.472 & 0.046 & 0.035 \\
\hline & & & & 5.5 & 0.505 & 0.050 & 0.029 \\
\hline & \multicolumn{2}{|c|}{$x=0.175$} & & 7.0 & 0.530 & 0.047 & 0.021 \\
\hline 1.625 & 0.868 & 0.060 & 0.030 & 9.0 & 0.443 & 0.057 & 0.015 \\
\hline 1.875 & 0.823 & 0.037 & 0.010 & 11.5 & 0.524 & 0.068 & 0.011 \\
\hline 2.25 & 0.829 & 0.026 & 0.008 & 15 & 0.525 & 0.056 & 0.007 \\
\hline 2.75 & 0.835 & 0.028 & 0.017 & 20 & 0.451 & 0.042 & 0.004 \\
\hline
\end{tabular}


TABLE 4 (CONTINUED)

\begin{tabular}{|c|c|c|c|c|c|c|c|}
\hline \multirow[t]{2}{*}{$Q^{2}$} & \multirow[t]{2}{*}{$F_{2}^{\mathrm{n}} / F_{2}^{\mathrm{p}}$} & \multicolumn{2}{|c|}{ Error } & \multirow[t]{2}{*}{$Q^{2}$} & \multirow[t]{2}{*}{$F_{2}^{\mathrm{n}} / F_{2}^{\mathrm{p}}$} & \multicolumn{2}{|c|}{ Error } \\
\hline & & stat. & syst. & & & stat. & syst. \\
\hline 27 & 0.535 & 0.045 & 0.003 & 65 & 0.427 & 0.101 & 0.002 \\
\hline 36 & 0.635 & 0.056 & 0.003 & 100 & 0.413 & 0.103 & 0.002 \\
\hline 48 & 0.557 & 0.062 & 0.002 & 160 & 0.286 & 0.247 & 0.001 \\
\hline 65 & 0.526 & 0.075 & 0.001 & & & & \\
\hline \multirow[t]{3}{*}{100} & 0.601 & 0.083 & 0.001 & \multirow{2}{*}{\multicolumn{4}{|c|}{$x=0.700$}} \\
\hline & & & & & & & \\
\hline & \multicolumn{2}{|c|}{$x=0.550$} & & 7.0 & 0.321 & 0.075 & 0.022 \\
\hline 5.5 & 0.374 & 0.070 & 0.038 & 9.0 & 0.350 & 0.091 & 0.016 \\
\hline 7.0 & 0.452 & 0.061 & 0.030 & 11.5 & 0.381 & 0.108 & 0.015 \\
\hline 9.0 & 0.496 & 0.084 & 0.022 & 15 & 0.390 & 0.090 & 0.011 \\
\hline 11.5 & 0.552 & 0.099 & 0.016 & 20 & 0.337 & 0.064 & 0.008 \\
\hline 15 & 0.311 & 0.069 & 0.010 & 27 & 0.278 & 0.061 & 0.006 \\
\hline 20 & 0.469 & 0.063 & 0.006 & 36 & 0.275 & 0.078 & 0.005 \\
\hline 27 & 0.448 & 0.061 & 0.004 & 48 & 0.332 & 0.096 & 0.004 \\
\hline 36 & 0.451 & 0.073 & 0.003 & 65 & 0.158 & 0.107 & 0.003 \\
\hline 48 & 0.566 & 0.092 & 0.002 & 100 & 0.500 & 0.152 & 0.002 \\
\hline
\end{tabular}

As a check of these results the ratio of beam fluxes $\Phi_{1} / \Phi_{2}$ (see eq. (5)) was extracted from the data as a function of $Q^{2}$ for each $x$ bin. The flux ratio was fitted with a linear function of $\ln Q^{2}$. The slopes determined from these fits (fig. $10 \mathrm{a})$ are consistent with zero for all $x$ bins with an average slope of $0.0003 \pm 0.0014$ (fig. 10b). This shows that the detector acceptance does not introduce a $Q^{2}$ dependence in the ratio $F_{2}^{\mathrm{n}} / F_{2}^{\mathrm{p}}$ (see subsect. 4.3).

TABLE 5

Results of the fits to the $Q^{2}$ dependence at fixed values of $x$

\begin{tabular}{lrcc}
\hline$x$ & $b$ & Fit probability & $\begin{array}{c}\text { Fit probability } \\
\text { for } b \text { set to zero }\end{array}$ \\
\hline 0.003 & $0.021 \pm 0.025$ & 0.29 & -0.32 \\
0.007 & $-0.004 \pm 0.011$ & 0.47 & 0.54 \\
0.015 & $-0.001 \pm 0.008$ & 0.59 & 0.65 \\
0.030 & $-0.002 \pm 0.007$ & 0.43 & 0.48 \\
0.050 & $0.012 \pm 0.008$ & 0.13 & 0.10 \\
0.080 & $-0.010 \pm 0.007$ & 0.13 & 0.10 \\
0.125 & $-0.024 \pm 0.007$ & 0.04 & $1 \times 10^{-3}$ \\
0.175 & $-0.043 \pm 0.008$ & 0.83 & $8 \times 10^{-4}$ \\
0.250 & $-0.029 \pm 0.008$ & 0.16 & $25 \times 10^{-4}$ \\
0.350 & $-0.032 \pm 0.011$ & $4 \times 10^{-3}$ & $4 \times 10^{-4}$ \\
0.450 & $0.032 \pm 0.018$ & 0.63 & 0.42 \\
0.550 & $0.010 \pm 0.027$ & 0.56 & 0.64 \\
0.700 & $-0.027 \pm 0.038$ & 0.75 & 0.78 \\
\hline
\end{tabular}


a)

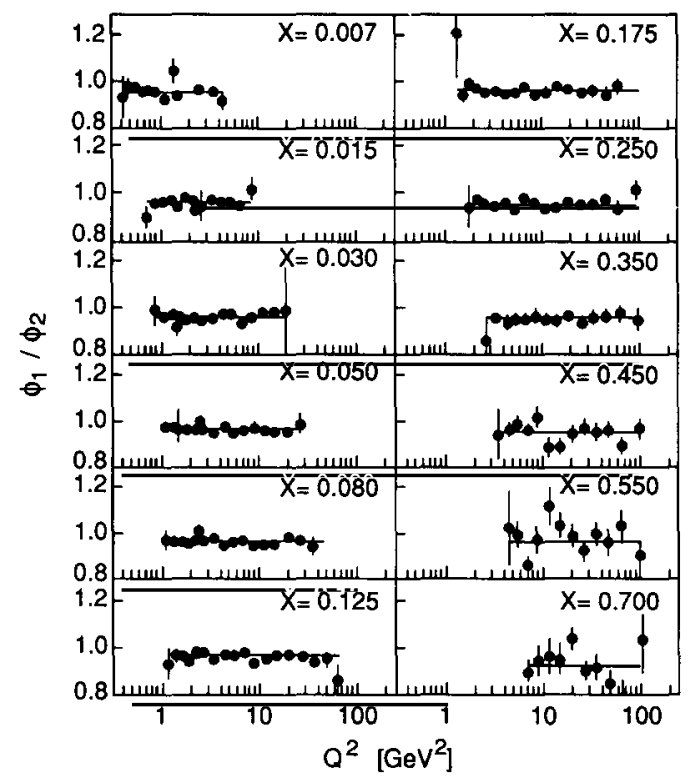

b)

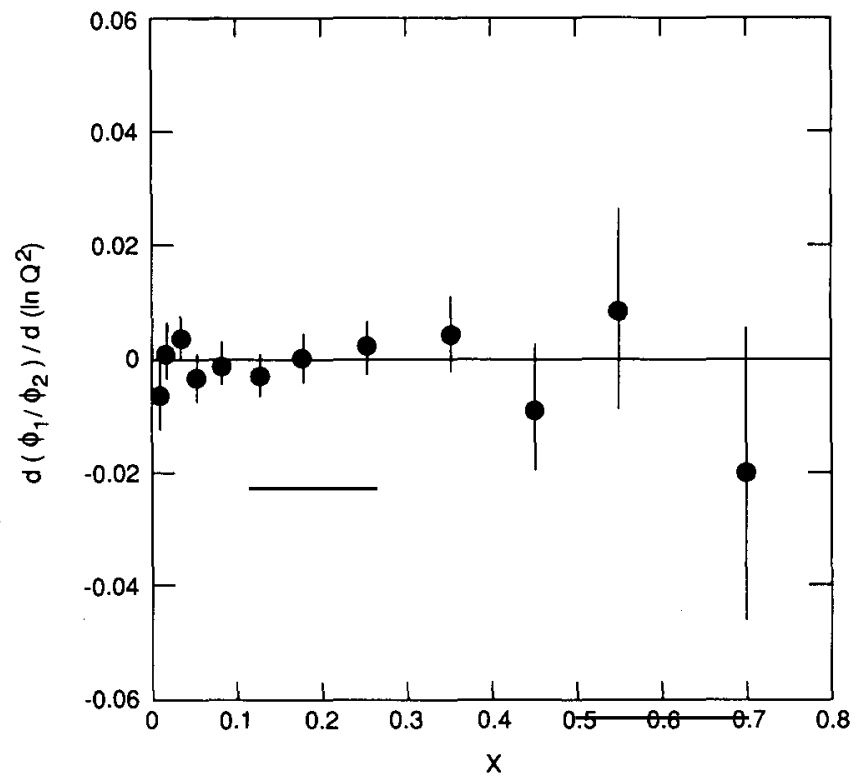

Fig. 10. (a) The flux ratio $\Phi_{1} / \Phi_{2}$ as a function of $Q^{2}$ for each $x$ bin: the lines represent fits of linear functions of $\ln Q^{2}$ to the data. (b) The derivative $\mathrm{d}\left(\Phi_{1} / \Phi_{2}\right) / \mathrm{d}\left(\ln Q^{2}\right)$ as a function of $x$. 


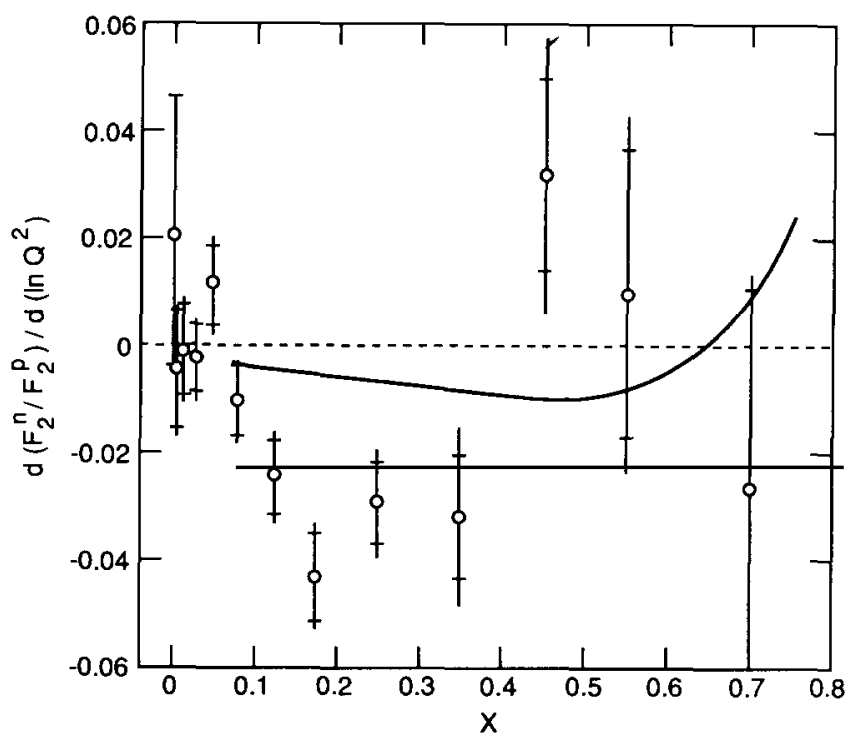

Fig. 11. The derivative $\mathrm{d}\left(F_{2}^{\mathrm{n}} / F_{2}^{\mathrm{p}}\right) / \mathrm{d}\left(\ln Q^{2}\right)$ as a function of $x$ for the data shown in fig. 9. The inner error bars represent the statistical uncertainties, the full bars the statistical and systematic uncertainties added in quadrature. The curve gives the result of a QCD calculation (see text).

In fig. 11 we show the fitted slope parameter $b$ as a function of $x$. The inner error bars represent the statistical errors and the full bars the quadratic sum of statistical and systematic errors. The latter were calculated by changing the ratio within each of its systematic uncertainties as listed in table 3 and adding in quadrature the resulting changes in slopes from all contributions. Also shown in this figure is a next-to-leading order QCD prediction [3] including target mass corrections [31]. This prediction is based on an analysis of the SLAC and BCDMS structure function data, which gives a value of the QCD scale parameter of $\Lambda=263 \mathrm{MeV}$ and a gluon distribution $x \cdot g(x)=2.60 \cdot(1-x)^{5.5}$, at $Q^{2}=20 \mathrm{GeV}^{2}$, for both the proton and neutron. For $x$ in the range $0.1-0.4$ the measured $Q^{2}$ dependence of $F_{2}^{\mathrm{n}} / F_{2}^{\mathrm{p}}$ is clearly different from the expectation of perturbative QCD. An interpretation of this difference in terms of higher-twist effects is discussed in sect. 6 .

\section{Higher-twist analysis}

In the QCD analysis of the SLAC and BCDMS data mentioned above, it was found that better descriptions of $F_{2}^{\mathrm{p}}$ and $F_{2}^{\mathrm{d}}$ were obtained after including 


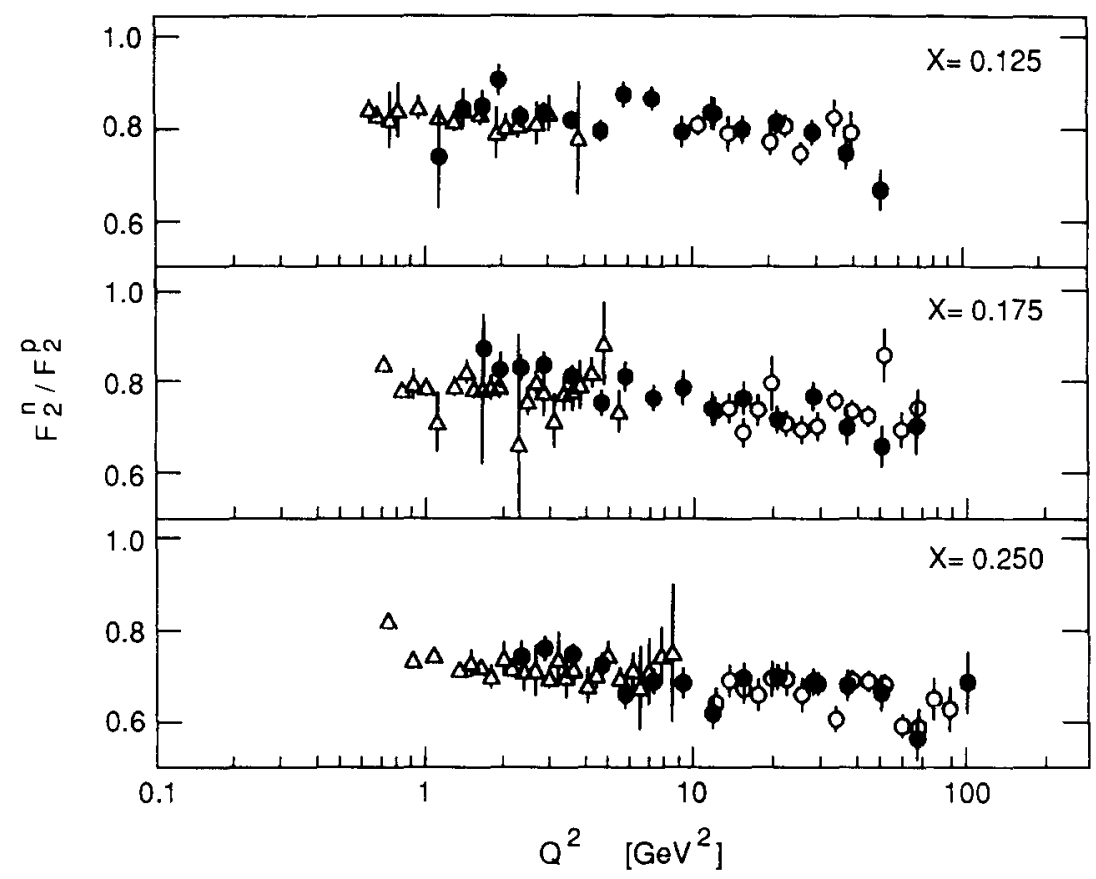

Fig. 12. The structure function ratio $F_{2}^{\mathrm{n}} / F_{2}^{\mathrm{p}}$ versus $Q^{2}$ in three $x$ bins which exhibit large $Q^{2}$ dependences. The present data (full circles) are given together with those from SLAC (triangles) and BCDMS (open circles). Only statistical errors are indicated.

phenomenological higher-twist terms. For this purpose, the structure functions $F_{2}$ were parametrised as

$$
F_{2}=F_{2}^{\mathrm{LT}}\left(1+\frac{C}{Q^{2}}\right)
$$

where the leading twist part $F_{2}^{\mathrm{LT}}$ obeys the next-to-leading order QCD evolution equations and includes target mass effects, and $C$ is the coefficient of the twist-four term. Using this parametrisation for $F_{2}$, and provided the coefficients $C^{\mathrm{p}}$ and $C^{\mathrm{n}}$ for proton and neutron are small, one has for the ratio:

$$
\frac{F_{2}^{\mathrm{n}}}{F_{2}^{\mathrm{p}}}=\left(\frac{F_{2}^{\mathrm{n}}}{F_{2}^{\mathrm{p}}}\right)^{\mathrm{LT}}\left(1-\frac{C^{\mathrm{p}}-C^{\mathrm{n}}}{Q^{2}}\right)
$$

The twist-four term will appear in $F_{2}^{\mathrm{n}} / F_{2}^{\mathrm{p}}$ only if the coefficients $C$ are different for the neutron and proton.

A comparison of the SLAC and BCDMS results and those from the present experiment shows that the structure function ratios as a function of $x$ and $Q^{2}$ found in the different experiments are compatible. This is illustrated in fig. 12 for 
three $x$ bins with the largest $Q^{2}$ dependence. In the following we used all this experimental information to extract the $Q^{2}$ dependence of $F_{2}^{\mathrm{n}} / F_{2}^{\mathrm{p}}$ with the smallest possible uncertainty.

The SLAC and BCDMS results on $F_{2}^{\mathrm{n}} / F_{2}^{\mathrm{p}}$ were obtained from separate measurements of the structure functions for hydrogen and deuterium. Their results are thus affected by overall normalisation uncertainties of $F_{2}^{\mathrm{d}}$ with respect to $F_{2}^{\mathrm{p}}$ of $1 \%$ (SLAC) and 2\% (BCDMS). Before combining all the data we therefore normalised the SLAC and BCDMS data for the ratio to those from the present experiment, which has a much smaller normalisation uncertainty. The changes in

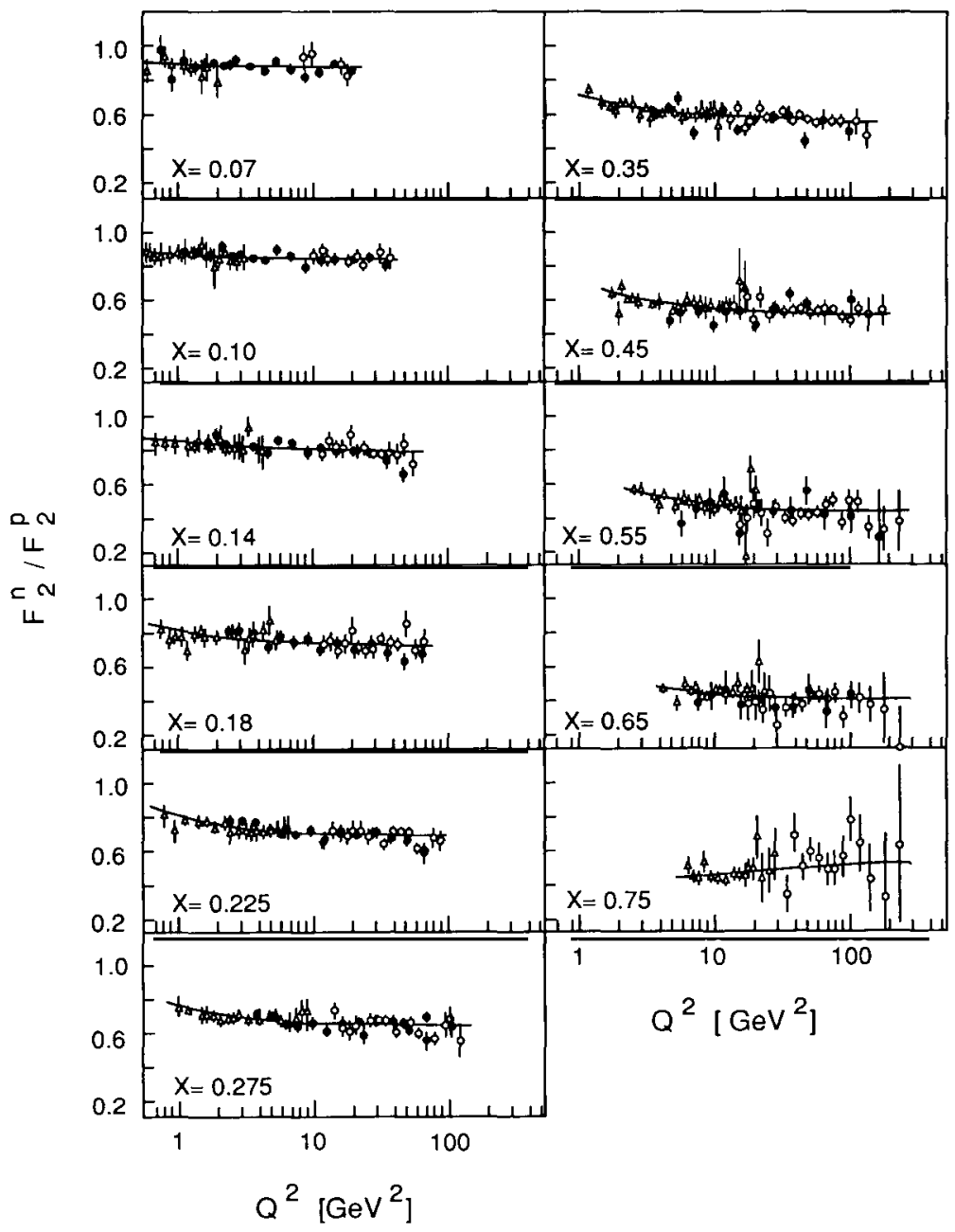

Fig. 13. The structure function ratio $F_{2}^{\mathrm{n}} / F_{2}^{\mathrm{p}}$ as a function of $Q^{2}$ for the present data (full circles) together with those from SLAC (triangles) and BCDMS (open circles) renormalised to the present data set. The curves represent fits used to extract the difference of twist-four coefficients for the proton and neutron (see text). 
TABLE 6

The difference of twist-four coefficients for the proton and neutron

\begin{tabular}{cccccc}
\hline$x$ & $\begin{array}{c}C^{\mathrm{p}}-\mathrm{C}^{\mathrm{n}} \\
(\mathrm{GeV})^{2}\end{array}$ & $\begin{array}{c}\text { Statistical } \\
\text { error }\end{array}$ & & \multicolumn{3}{c}{ Systematic error } \\
\cline { 5 - 6 } & & 0.024 & 0.006 & 0.157 & 0.157 \\
0.070 & -0.001 & 0.019 & 0.009 & 0.132 & 0.132 \\
0.100 & -0.009 & 0.023 & 0.014 & 0.094 & 0.095 \\
0.140 & -0.039 & 0.026 & 0.017 & 0.055 & 0.057 \\
0.180 & -0.062 & 0.025 & 0.017 & 0.017 & 0.024 \\
0.225 & -0.125 & 0.031 & 0.023 & 0.004 & 0.023 \\
0.275 & -0.099 & 0.033 & 0.033 & 0.000 & 0.033 \\
0.350 & -0.194 & 0.063 & 0.049 & 0.000 & 0.049 \\
0.450 & -0.147 & 0.11 & 0.074 & 0.000 & 0.074 \\
0.550 & -0.44 & 0.31 & 0.13 & 0.000 & 0.13 \\
0.650 & -0.56 & 0.75 & 0.21 & 0.000 & 0.21 \\
0.750 & 0.06 & & & & total \\
\hline
\end{tabular}

the normalisation were determined from the regions of kinematic overlap with our data and correspond to a $-(0.6 \pm 0.4) \%$ change for the SLAC measurement of $F_{2}^{p}$ and $-(0.8 \pm 0.4) \%$ for that of BCDMS. A phenomenological parametrisation of the three data sets after this renormalisation is given in appendix $B$.

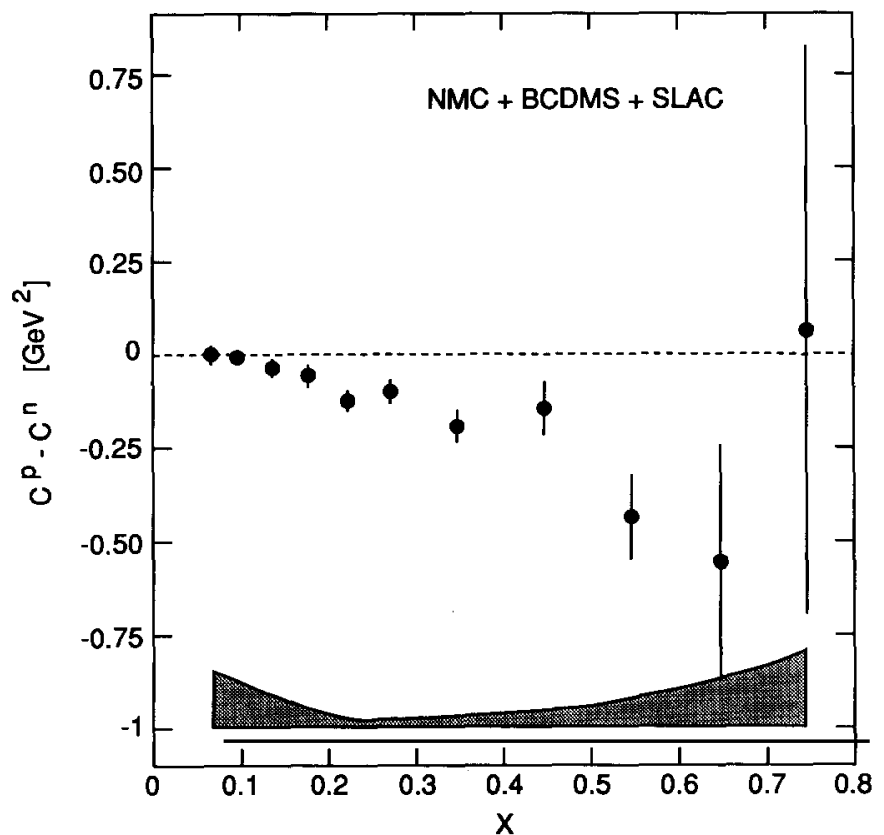

Fig. 14. The difference between twist-four coefficients for the proton and neutron obtained from the NMC and renormalised SLAC and BCDMS data. The statistical uncertainties are indicated with error bars, the systematic uncertainty by the error band. 
We determined the difference $C^{\mathrm{p}}-C^{\mathrm{n}}$ from a fit of eq. (8) to the NMCSLAC-BCDMS data keeping $\left(F_{2}^{\mathrm{n}} / F_{2}^{\mathrm{p}}\right)^{\mathrm{LT}}$ fixed. This ratio was obtained from the QCD analysis mentioned in sect. 5, for $x>0.07$. The sensitivity of $\left(F_{2}^{\mathrm{n}} / F_{2}^{\mathrm{p}}\right)^{\mathrm{LT}}$ to the value of $\Lambda$ is small. The result of the fit is presented in fig. 13 in the $x$ bins used in this QCD analysis. The fit gives a good simultaneous description of the three data sets $\left(\chi^{2} /\right.$ d.f. $\left.=371 / 465\right)$. The corresponding values for $C^{\mathrm{p}}-C^{\mathrm{n}}$ are listed in table 6 and shown in fig. 14. This result is insensitive to the gluon distribution provided it is the same for the proton and neutron. However, a difference of 1 between the exponents of the proton and neutron gluon distributions leads to a significant change of the result at $x<0.2$. This change was taken to be an estimate of the systematic error. At larger $x$ the dominant source is the uncertainty on the normalisation of the SLAC and BCDMS data with respect to those of NMC. Fig. 14 shows that the twist-four coefficients are significantly smaller for the proton than for the neutron for $x$ larger than 0.2 .

Similar fits to the three data sets were made with the normalisation of the SLAC and BCDMS data left free, instead of being fixed to the NMC data in the overlap regions, and give comparable results. The use of another parametrisation of twist-four terms, $F_{2}=F_{2}^{\mathrm{LT}}+C / Q^{2}$, also leads to the conclusion that a significant difference exists between the proton and the neutron twist-four coefficients.

\section{Summary}

We have obtained the ratio of neutron and proton structure functions, $F_{2}^{\mathrm{n}} / F_{2}^{\mathrm{p}}$, from simultaneous measurements of deep inelastic muon scattering on hydrogen and deuterium at incident energies of 90 and $280 \mathrm{GeV}$. The data cover the kinematic range $x=0.002-0.80$ and $Q^{2}=0.1-190 \mathrm{GeV}^{2}$ and have small systematic errors. The $x$ dependence of the ratio, averaged over $Q^{2}$, shows no evidence of sizeable shadowing in deuterium. The ratio measured at the lowest value of $x$ is consistent with unity.

In the intermediate- $x$ range $(0.1-0.4)$ the observed $Q^{2}$ dependence of the ratio is stronger than predicted by perturbative QCD and target mass effects. This difference can be attributed to different higher-twist contributions for the proton and neutron. The difference of twist-four coefficients was extracted from an analysis of NMC, SLAC and BCDMS data on $F_{2}^{\mathrm{n}} / F_{2}^{\mathrm{p}}$. This analysis shows that the twist-four coefficient for the proton is significantly lower than that for the neutron for $x$ larger than 0.2 .

\section{Appendix A}

The deuterium structure function used in the radiative correction procedure was obtained from a fit to deep inelastic scattering data including those in the $\Delta(1232)$ resonance region (see subsect. 4.5 ). 
For this purpose, the structure function was parametrised as

$$
F_{2}^{\mathrm{d}}\left(x, Q^{2}\right)=\left[1-G^{2}\left(Q^{2}\right)\right]\left[F^{\mathrm{dis}}\left(x, Q^{2}\right)+F^{\mathrm{res}}\left(x, Q^{2}\right)+F^{\mathrm{bg}}\left(x, Q^{2}\right)\right],
$$

where $F^{\text {dis }}$ and $F^{\text {res }}$ are the contributions from the deep inelastic and resonance regions respectively and $F^{\text {bg }}$ describes the background under the resonance. The nucleon electromagnetic form factor is $G\left(Q^{2}\right)=\left(1+Q^{2} / 0.71\right)^{-2}$ : the term $1-G^{2}$ in eq. (A.1) suppresses $F_{2}$ at low values of $Q^{2}$ where elastic scattering on the nucleon dominates.

The contribution from the deep inelastic region was parametrised as

$$
F^{\mathrm{dis}}\left(x, Q^{2}\right)=\left[\frac{5}{18} \frac{3}{B\left(\eta_{1}, \eta_{2}+1\right)} x_{\mathrm{w}}^{\eta_{1}}\left(1-x_{\mathrm{w}}\right)^{\eta_{2}}+\frac{1}{3} \eta_{3}\left(1-x_{\mathrm{w}}\right)^{\eta_{4}}\right] S\left(x, Q^{2}\right)
$$

where $x_{\mathrm{w}}=\left(Q^{2}+m_{\mathrm{a}}^{2}\right) /\left(2 M \nu+m_{\mathrm{b}}^{2}\right)$ with $m_{\mathrm{a}}^{2}=0.351 \mathrm{GeV}^{2}$ and $m_{\mathrm{b}}^{2}=1.512 \mathrm{GeV}^{2}$. The quantity $B$ is Euler's beta function and $\eta_{1}, \ldots, \eta_{4}$ are linear functions of the variable $\bar{s}$,

$$
\eta_{i}=\alpha_{i}+\beta_{i} \bar{s}
$$

where

$$
\bar{s}=\ln \frac{\ln \left[\left(Q^{2}+m^{2}\right) / \Lambda^{2}\right]}{\ln \left[\left(Q_{0}^{2}+m_{\mathrm{a}}^{2}\right) / \Lambda^{2}\right]}
$$

with $Q_{0}^{2}=2.0 \mathrm{GeV}^{2}$ and $\Lambda=0.2 \mathrm{GeV}$. The constants $\alpha_{1}, \ldots, \alpha_{4}$ and $\beta_{1}, \ldots, \beta_{4}$ were free parameters in the fit.

The term $S\left(x, Q^{2}\right)$ in eq. (A.2) suppresses $F^{\text {dis }}$ in the resonance region close to the single pion production threshold:

$$
S\left(x, Q^{2}\right)=1-\mathrm{e}^{-a\left(W-w_{\mathrm{thr}}\right)}
$$

with $W^{2}=M^{2}+2 M \nu-Q^{2}, W_{\mathrm{thr}}=1.03 \mathrm{GeV}$ and $a=4.177 \mathrm{GeV}^{-1}$

The form adopted for the contribution from the resonance region was

$$
F^{\mathrm{res}}\left(x, Q^{2}\right)=\alpha_{5}^{2} G^{3 / 2} \mathrm{e}^{-\left(W-m_{\lrcorner}\right)^{2} / \Gamma^{2}}
$$

with $m_{\Delta}=1.232 \mathrm{GeV}, \Gamma=0.0728 \mathrm{GeV}$ and $\alpha_{5}$ is a free parameter in the fit. This parametrisation takes into account only the $\Delta(1232)$ contribution; higher mass resonances are neglected. 
TABLE A.1

Parameters extracted from the fit to $F_{2}^{\text {d }}$

\begin{tabular}{lccccc}
\hline & \multicolumn{1}{c}{$\alpha_{1}$} & $\beta_{1}$ & $\alpha_{2}$ & $\beta_{2}$ & $\alpha_{3}$ \\
\hline Standard & 0.75966 & -0.18202 & 3.5200 & 0.46256 & 0.83691 \\
Lower limit & 0.74296 & -0.20019 & 3.4819 & 0.45823 & 0.79157 \\
Upper limit & 0.77171 & -0.19672 & 3.5390 & 0.40757 & 0.94675 \\
\hline & $\beta_{3}$ & $\alpha_{4}$ & $\beta_{4}$ & $\alpha_{5}$ & $\alpha_{6}$ \\
\hline Standard & 0.97906 & 12.876 & -2.9558 & 0.89456 & 0.16452 \\
Lower limit & 0.96662 & 13.247 & -3.5632 & 0.89456 & 0.16452 \\
Upper limit & 1.0889 & 13.352 & -3.9720 & 0.89456 & 0.16452 \\
\hline
\end{tabular}

See subsect. 4.5 for the definition of lower and upper limits. The parameters $\alpha_{5}$ and $\alpha_{6}$ were kept fixed to their values from the standard fit.

The background under the resonance region was parametrised as

$$
F^{\mathrm{bg}}\left(x, Q^{2}\right)=\alpha_{6}^{2} G^{1 / 2} \xi \mathrm{e}^{-b\left(W-W_{\mathrm{thr}}\right)^{2}}
$$

where

$$
\xi=\sqrt{\frac{\left[(W+c)^{2}+M^{2}-m_{\pi}^{2}\right]^{2}}{4(W+c)^{2}}-M^{2}}
$$

with $b=0.5 \mathrm{GeV}^{-1}$ and $c=0.05 \mathrm{GeV}$. The parameter $\alpha_{6}$ was left free in the fit.

The fitted parameters are listed in table A.1. The $\chi^{2}$ of the fit is 1975 for 595 degrees of freedom. This poor $\chi^{2}$ is due to the fact that the data were weighted only by their statistical errors: systematic uncertainties were not taken into account.

\section{Appendix B}

After renormalisation of the data on $F_{2}^{\mathrm{p}}$ from SLAC by $-0.6 \%$ and BCDMS by $-0.8 \%$ (see text), the following parametrisation gives a good description of the three data sets (NMC, SLAC, BCDMS):

$$
F_{2}^{\mathrm{n}} / F_{2}^{\mathrm{p}}\left(x, Q^{2}\right)=A(x)\left(Q^{2} / 20\right)^{B(x)}\left(1+x^{2} / Q^{2}\right)
$$

where

$$
\begin{aligned}
& A(x)=0.979-1.692 x+2.797 x^{2}-4.313 x^{3}+3.075 x^{4} \\
& B(x)=-0.171 x+0.244 x^{2}
\end{aligned}
$$


The $\chi^{2}$ of this fit is 475 for 571 degrees of freedom. The parametrisation is valid in the kinematic domain of the three data sets.

\section{References}

[1] A.J. Buras, Rev. Mod. Phys. 52 (1980) 199; G. Curci, W. Furmański and R. Petronzio, Nucl. Phys. B175 (1980) 27; W. Furmański and R. Petronzio, Phys. Lett. B97 (1980) 437

[2] R.L. Jaffe and M. Soldate, Phys. Lett. B105 (1981) 467; P. Castorina and P.J. Mulders, Phys. Rev. D31 (1985) 2753

[3] A. Milsztajn and M. Virchaux, Saclay report DPhPE 91-08 (1991), submitted to Phys. Lett. B

[4] EMC Collaboration, J.J. Aubert et al., Nucl. Phys. B293 (1987) 740

[5] S. Stein et al., Phys. Rev. D12 (1975) 1884

[6] A. Bodek et al., Phys. Rev. D20 (1979) 1471

[7] M.D. Mestayer et al., Phys. Rev. D27 (1983) 285

[8] BCDMS Collaboration, A.C. Benvenuti et al., Phys. Lett. B237 (1990) 599

[9] L.W. Whitlow, PhD thesis, SLAC-Report-357(1990)

[10] NMC Collaboration, D. Allasia et al., Phys. Lett. B249 (1990) 366

[11] NMC Collaboration, P. Amaudruz et al., Phys. Rev. Lett. 66 (1991) 2712

[12] L.W. Whitlow et al., Phys. Lett. B250 (1990) 193

[13] EMC Collaboration, O.C. Allkofer et al., Nucl. Instrum. Methods 179 (1981) 445; EMC Collaboration, J.P. Albanese et al., Nucl. Instrum. Methods 212 (1983) 111; EMC Collaboration, J.J. Aubert et al,, Nucl. Phys. B259 (1985) 189

[14] Review of Particle Properties, Phys. Lett. B223 (1990)

[15] F.L. Navarria et al., Nucl. Instrum. Methods 212 (1983) 125

[16] L.W. Mo and Y.S. Tsai, Rev. Mod. Phys. 41 (1969) 205; Y.S. Tsai, SLAC-PUB-848 (1971)

[17] M.D. Mestayer, PhD thesis, SLAC-REPORT-214 (1978)

[18] BCDMS Collaboration, A.C. Benvenuti et al., Phys. Lett. B223 (1989) 485; BCDMS Collaboration, A.C. Benvenuti et al., Phys. Lett. B237 (1990) 592

[19] EMC Collaboration, M. Arneodo et al., Nucl. Phys. B333 (1990) 1

[20] CHIO Collaboration, B.A. Gordon et al., Phys. Rev. D20 (1979) 2645

[21] G. Höhler et al., Nucl. Phys. B114 (1976) 505

[22] J. Bernabeu and P. Pascual, Nuovo Cimento 10A (1972) 61; J. Bernabeu, Nucl. Phys. B49 (1972) 186

[23] M.P. Locher and A. Švarc, Z. Phys. A338 (1991) 89

[24] A.A. Akhundov et al., Sov. J. Nucl. Phys. 44 (1986) 988; D.Yu. Bardin and N.M. Shumeiko, Sov. J. Nucl. Phys. 29 (1979) 499; A.A. Akhundov et al., Sov. J. Nucl. Phys. 26 (1977) 660

[25] C. Lietzke and S.J. Wimpenny, Univ. of California (Riverside) report UCR/DIS-89-06

[26] H. Engelien, PhD thesis, Freiburg University (1991)

[27] W.B. Atwood, PhD thesis, SLAC-REPORT-185 (1975)

[28] H. Arenhövel, private communication

[29] P.N. Harriman et al., Phys. Rev. D42 (1990) 798

[30] B. Badełek and J. Kwieciński, Warsaw Univ. preprint IFD/3/91 (1991), Nucl. Phys, B370 (1992) 278

N.N. Nikolaev and B.G. Zakharov, Z. Phys. C49 (1991) 607;

U.R. Zoller, Inst. of Theoretical and Experimental Physics, Moscow preprint 58/91

[31] H. Georgi and H.D. Politzer, Phys. Rev. D14 (1976) 1829 